\title{
On the Sum Rate of Gaussian Multiterminal Source Coding: New Proofs and Results
}

\author{
Jia Wang, Jun Chen, Member, IEEE, and Xiaolin Wu, Senior Member, IEEE
}

\begin{abstract}
We show that the lower bound on the sum rate of the direct and indirect Gaussian multiterminal source coding problems can be derived in a unified manner by exploiting the semidefinite partial order of the distortion covariance matrices associated with the minimum mean squared error (MMSE) estimation and the so-called reduced optimal linear estimation, through which an intimate connection between the lower bound and the Berger-Tung upper bound is revealed. We give a new proof of the minimum sum rate of the indirect Gaussian multiterminal source coding problem (i.e., the Gaussian CEO problem). For the direct Gaussian multiterminal source coding problem, we derive a general lower bound on the sum rate and establish a set of sufficient conditions under which the lower bound coincides with the Berger-Tung upper bound. We show that the sufficient conditions are satisfied for a class of sources and distortion constraints; in particular, they hold for arbitrary positive definite source covariance matrices in the high-resolution regime. In contrast with the existing proofs, the new method does not rely on Shannon's entropy power inequality.
\end{abstract}

Index Terms-CEO problem, entropy power inequality, minimum mean squared error (MMSE), multiterminal source coding, semidefinite programming.

\section{INTRODUCTION}

D IRECT and indirect multiterminal source coding are two fundamental problems in network information theory.

The indirect version, also known as the CEO problem (see Fig. 1), was formulated in [2]. The quadratic Gaussian case of the CEO problem, first studied by Viswanathan and Berger [21], has received particular attention. Major progress on this problem was made by Oohama in [6]. The rate-distortion region of the Gaussian CEO problem was completely characterized in [7] and [13]. The main technique developed in [6] and later refined in [7] and [13] is the application of Shannon's entropy power inequality to relate various information quantities. A short survey on the Gaussian CEO problem can be found in [10].

Direct multiterminal source coding (see Fig. 2), which will be referred to simply as multiterminal source coding, is a lossy

Manuscript received April 09, 2009; accepted January 28, 2010. Date of current version July 14,2010 . The work of J. Wang was supported by the NSFC by Grant 60802020 and by the 973 Program (2010CB731401, 2010CB731406).

The material in this paper was presented in part at the IEEE International Symposium on Information Theory, Seoul, Korea, June-July 2009.

J. Wang is with the Department of Electronic Engineering, Shanghai Jiao Tong University, Shanghai 200240, China (e-mail: jiawang@ sjtu.edu.cn).

J. Chen and X. Wu are with the Department of Electrical and Computer Engineering, McMaster University, Hamilton, ON L8S 4K1, Canada (e-mail: junchen@ece.mcmaster.ca; xwu@ece.mcmaster.ca).

Communicated by E. Ordentlich, Associate Editor for Source Coding.

Digital Object Identifier 10.1109/TIT.2010.2050960 extension of the Slepian-Wolf coding problem [16]. Significant effort has been devoted to characterizing the rate-distortion region for this problem. The best inner bound, given in [1] and [20], is often referred to as the Berger-Tung bound. For the Gaussian two-terminal case, Wagner et al. [22], building upon the work by Oohama [5], proved that the Berger-Tung inner bound is tight. Notably, they derived the minimum sum rate of the Gaussian two-terminal source coding problem by coupling it to a Gaussian CEO problem, therefore, made an implicit use of Shannon's entropy power inequality.

There are several extensions [8], [9], [11], [12], [18], [19] to the aforementioned results. In particular, Tavilder et al. observed in [18] that Shannon's entropy power inequality can be partially replaced by the worst additive noise lemma by Diggavi and Cover [4] in the derivation of the minimum sum rate of the Gaussian CEO problem.

We shall present a new approach for deriving the lower bound on the sum rate of the Gaussian multiterminal source coding problems. The key ingredient is a simple fact about the semidefinite partial order of the distortion covariance matrices associated with the MMSE estimation and the so-called reduced optimal linear estimation. Due to its estimation-theoretic nature, the new approach does not require Shannon's entropy power inequality. In addition to its technical simplicity, the new approach is also conceptually appealing in the sense that it makes the connection between the lower bound and the Berger-Tung upper bound transparent. As a consequence, we are able to give an alternative proof of the minimum sum rate of the Gaussian CEO problem. The new approach also leads to a general lower bound on the sum rate of the Gaussian multiterminal source coding problem.

The remainder of this paper is organized as follows. In Section II, we introduce the MMSE estimation and the reduced optimal linear estimation with an emphasis on the semidefinite partial order of the distortion covariance matrices associated with them. A new proof of the minimum sum rate of the Gaussian CEO problem is given in Section III. In Section IV, we derive a general lower bound on the sum rate of the Gaussian multiterminal source coding problem and establish a set of sufficient conditions under which the lower bound is tight. We show that the sufficient conditions are satisfied for a class of sources and distortion constraints; in particular, they hold for arbitrary positive definite source covariance matrices in the high-resolution regime. Section V contains some concluding remarks.

We typically use uppercase letters for random variables and boldfaced letters for matrices. There are a few exceptions, which should be clear from the context. We use $\mathbf{I}, \mathbf{0}$, and $\mathbf{1}$ to denote the identity matrix, the all-zero matrix (not necessarily a square matrix), and the all-one column vector, respectively. For simplicity, 


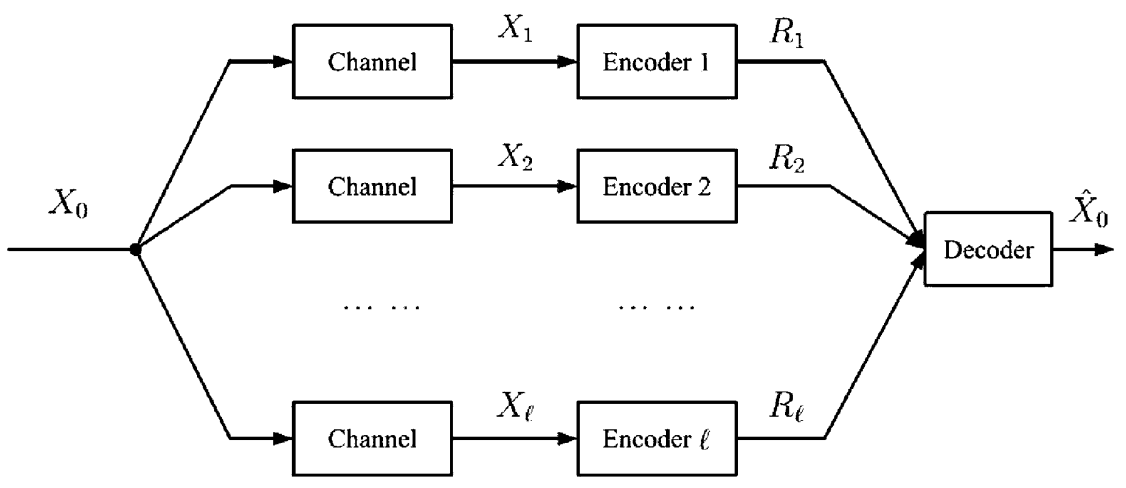

Fig. 1. Indirect multiterminal source coding (the CEO problem).

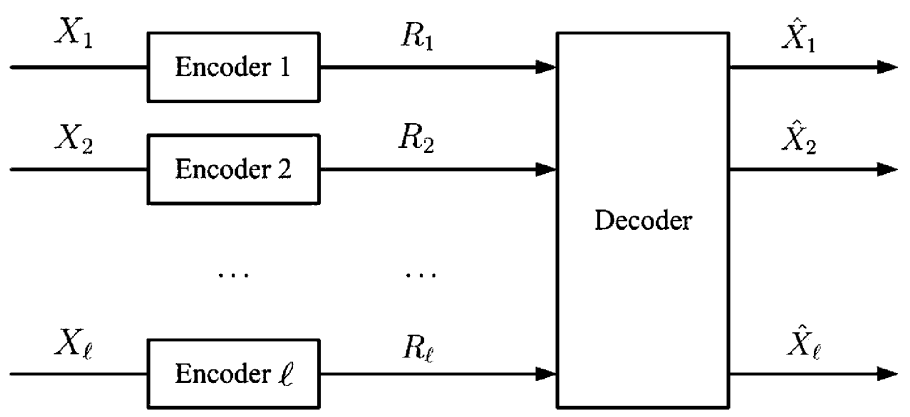

Fig. 2. Direct multiterminal source coding.

sometimes we write the row vector $(Z(1), Z(2), \ldots, Z(n))$ as $Z^{n}$. Throughout this paper, we assume the logarithm function is to base $e$.

\section{PRELIMINARIES}

Throughout this section, we assume that the random objects are of mean zero.

Let $\mathbf{X}$ be an $\ell \times n$ random matrix. Note that the covariance matrix of $\mathbf{X}$ has totally $(\ell \times n)^{2}$ elements. It turns out that the following notion of reduced covariance matrix suffices for our purpose. Specifically, we define the reduced covariance matrix of $\mathbf{X}$ as

$$
\boldsymbol{\Sigma}_{\mathbf{X}}=\frac{1}{n} \mathbb{E}\left[\mathbf{X X}^{T}\right]
$$

It is clear that $\boldsymbol{\Sigma}_{\mathbf{X}}$ is an $\ell \times \ell$ positive semidefinite matrix. Alternatively, we can write

$$
\boldsymbol{\Sigma}_{\mathbf{X}}=\frac{1}{n} \sum_{i=1}^{n} \mathbb{E}\left[\mathbf{X}(i) \mathbf{X}(i)^{T}\right]
$$

where $\mathbf{X}(i)$ is the $i$ th column of $\mathbf{X}$. Therefore, the reduced covariance matrix of $\mathbf{X}$ is given by averaging the covariance matrices of the column vectors of $\mathbf{X}$; in particular, it coincides with the conventional covariance matrix when $\mathbf{X}$ is a column vector. More generally, we define

$$
\boldsymbol{\Sigma}_{\mathbf{X Y}}=\frac{1}{n} \mathbb{E}\left[\mathbf{X Y} \mathbf{Y}^{T}\right]
$$

for any random matrices $\mathbf{X}$ and $\mathbf{Y}$ with the same column dimension $n$.

The following lemma states a well-known fact about the MMSE estimation (i.e., the conditional expectation) [17].
Lemma 1: For any random objects $X^{n}$ and $\omega$, we have

$$
\mathbb{E}\left[\left(X^{n}-\mathbb{E}\left[X^{n} \mid \omega\right]\right)^{T} Y^{m}(\omega)\right]=\mathbf{0}
$$

where $Y^{m}(\omega)$ is an arbitrary measurable function of $\omega$.

We use $\prec(\prec)$ to denote positive semidefinite (definite) partial ordering. Specifically, for two matrices $\mathbf{A}$ and $\mathbf{B}, \mathbf{A} \preceq(\prec$ B means $\mathbf{B}-\mathbf{A}$ is positive semidefinite (definite). Note that $\mathbf{A}(\preceq) \prec \mathbf{B}$ degrades to $\mathbf{A} \leq(<) \mathbf{B}$ if $\mathbf{A}$ and $\mathbf{B}$ are $1 \times 1$ matrices.

The following result is a simple consequence of Lemma 1.

Lemma 2: For any random object $\omega$ and an $\ell \times n$ random matrix $\mathbf{X}$, we have

$$
\boldsymbol{\Sigma}_{\mathbf{X}-\mathbb{E}[\mathbf{X} \mid \omega]} \preceq \boldsymbol{\Sigma}_{\mathbf{X}-\mathbf{Y}(\omega)}
$$

where $\mathbf{Y}(\omega)$ is an arbitrary $\ell \times n$ matrix determined by $\omega$.

Define $\mathbf{D}_{\mathbf{X} \mid \omega}=\boldsymbol{\Sigma}_{\mathbf{X}-\mathbb{E}[\mathbf{X} \mid \omega]}$. We shall refer to $\mathbf{D}_{\mathbf{X} \mid \omega}$ as the distortion covariance matrix of the MMSE estimation for $\mathbf{X}$ given $\omega$.

For an $\ell \times n$ random matrix $\mathbf{X}$ and an $m \times n$ random matrix $\mathbf{Y}$, we denote the $i$ th row of $\mathbf{X}$ and $\mathbf{Y}$ by $X_{i}^{n}$ and $Y_{i}^{n}$, respectively. The reduced optimal linear estimation of $\mathbf{X}$ given $\mathbf{Y}$ is $\mathbf{B}^{*} \mathbf{Y}$, where $\mathbf{B}^{*}=\left(b_{i, j}^{*}\right)$ is an $\ell \times m$ matrix specified by the solution to the following optimization problem:

$\min _{\left(b_{i, j}\right) \in \mathbb{R}^{\ell \times m}} \mathbb{E}\left[\left(X_{i}^{n}-\sum_{j=1}^{m} b_{i, j} Y_{j}^{n}\right)\right.$

$$
\left.\times\left(X_{i}^{n}-\sum_{j=1}^{m} b_{i, j} Y_{j}^{n}\right)^{T}\right] .
$$


Define $\tilde{\mathbf{D}}_{\mathbf{X} \mid \mathbf{Y}}=\boldsymbol{\Sigma}_{\mathbf{X}-\mathbf{B}^{*} \mathbf{Y}}$. We shall refer to $\tilde{\mathbf{D}}_{\mathbf{X} \mid \mathbf{Y}}$ as the distortion covariance matrix of the reduced optimal linear estimation for $\mathbf{X}$ given $\mathbf{Y}$. It is easy to verify that

$$
\begin{aligned}
\mathbf{B}^{*} & =\boldsymbol{\Sigma}_{\mathbf{X Y}} \boldsymbol{\Sigma}_{\mathbf{Y}}^{-1} \\
\tilde{\mathbf{D}}_{\mathbf{X} \mid \mathbf{Y}} & =\boldsymbol{\Sigma}_{\mathbf{X}}-\boldsymbol{\Sigma}_{\mathbf{X Y}} \boldsymbol{\Sigma}_{\mathbf{Y}}^{-1} \boldsymbol{\Sigma}_{\mathbf{X Y}}^{T} .
\end{aligned}
$$

More generally, given random matrices $\mathbf{Y}_{1}, \mathbf{Y}_{2}, \ldots, \mathbf{Y}_{k}$ with dimensions $m_{i} \times n, i=1,2, \ldots, k$, we define

$$
\tilde{\mathbf{D}}_{\mathbf{X} \mid \mathbf{Y}_{1}, \mathbf{Y}_{2}, \ldots, \mathbf{Y}_{k}}=\tilde{\mathbf{D}}_{\mathbf{X} \mid \mathbf{Y}}
$$

where $\mathbf{Y}$ is a $\left(\sum_{i=1}^{k} m_{i}\right) \times n$ random matrix constructed by juxtaposing $\mathbf{Y}_{1}, \mathbf{Y}_{2}, \ldots, \mathbf{Y}_{l}$, i.e.,

$$
\mathbf{Y}=\left(\begin{array}{c}
\mathbf{Y}_{1} \\
\vdots \\
\mathbf{Y}_{k}
\end{array}\right)
$$

The following result is a direct consequence of Lemma 2. It reveals the semidefinite partial order of the distortion covariance matrices associated with the MMSE estimation and the reduced optimal linear estimation.

\section{Lemma 3: $\mathbf{D}_{\mathbf{X} \mid \mathbf{Y}} \preceq \tilde{\mathbf{D}}_{\mathbf{X} \mid \mathbf{Y}}$.}

It will be seen that Lemma 3 induces the constraints in the semidefinite programming bounds for the indirect and direct Gaussian multiterminal source coding problems, which, in a sense, provides a unified framework for proving the converse theorem of both problems.

\section{The Gaussian CEO PRoblem}

We shall first use the Gaussian CEO problem as a simple example to illustrate the new method. Consider a remote Gaussian source $X_{0}$ with mean zero and positive variance $\sigma_{0}^{2}$. Let $X_{i}=$ $X_{0}+N_{i}, i=1,2, \ldots, \ell$, where $N_{i}, i=1,2, \ldots, \ell$, are zeromean Gaussian random variables with positive variances $\sigma_{i}^{2}$, $i=1,2, \ldots, \ell$, respectively. We assume $X_{0}, N_{1}, \ldots, N_{\ell}$ are independent. It is known that $X_{0}$ can be expressed as

$$
X_{0}=Y+N
$$

where

$$
Y=\mathbb{E}\left[X_{0} \mid X_{1}, X_{2}, \ldots, X_{\ell}\right]=\sum_{i=1}^{\ell} \frac{\sigma_{N}^{2}}{\sigma_{i}^{2}} X_{i}
$$

$\left(X_{1}, X_{2}, \ldots, X_{\ell}\right)$ with variance and $N$ is a zero-mean Gaussian random variable independent of

$$
\sigma_{N}^{2}=\left(\frac{1}{\sigma_{0}^{2}}+\sum_{i=1}^{\ell} \frac{1}{\sigma_{i}^{2}}\right)^{-1} .
$$

Let $\left\{\left(X_{0}(t), X_{1}(t), \ldots, X_{\ell}(t), Y(t), N(t)\right)\right\}_{t=1}^{\infty}$ be i.i.d. copies of $\left(X_{0}, X_{1}, \ldots, X_{\ell}, Y, N\right)$.

Definition 1: We say sum rate $R$ is achievable with respect to distortion constraint $d$ if there exist $\ell$ encoding functions $f_{i}$ : $\mathbb{R}^{n} \rightarrow\left\{1,2, \ldots, M_{i}\right\}, i=1,2, \ldots, \ell$, such that

$$
\begin{aligned}
\frac{1}{n} \sum_{i=1}^{l} \log M_{i} & \leq R \\
\mathbf{D}_{X_{0}^{n} \mid W_{\mathcal{L}}} & \leq d,
\end{aligned}
$$

where $W_{\mathcal{L}}=\left(W_{1}, W_{2}, \ldots, W_{\ell}\right)^{T}$ with $W_{i}=f_{i}\left(X_{i}^{n}\right), i=$ $1,2, \ldots, \ell$. The minimum sum rate $R^{\star}(d)$ is the infimum of all achievable sum rates with respect to distortion constraint $d$.

Remark: It is clear that we can restrict our attention to $d \in$ $\left(\sigma_{N}^{2}, \sigma_{0}^{2}\right]$.

\section{A. Lower Bound}

Let $f_{i}: \mathbb{R}^{n} \rightarrow\left\{1,2, \ldots, M_{i}\right\}, i=1,2, \ldots, \ell$, be $\ell$ encoding functions such that $\mathbf{D}_{X_{0}^{n} \mid W_{\mathcal{L}}} \leq d$. Define $d_{0}=\mathbf{D}_{X_{0}^{n} \mid W_{\mathcal{L}}}$ and $d_{i}=\mathbf{D}_{X_{i}^{n} \mid X_{0}^{n}, W_{i}}, i=1,2, \ldots, \ell$. Without loss of generality, we assume $d_{0} \in\left(\sigma_{N}^{2}, d\right]$ and $d_{i} \in\left(0, \sigma_{i}^{2}\right]$ for $i=1,2, \ldots, \ell$.

First we shall derive a lower bound on the sum rate and express it as a function of $d_{0}, d_{1}, \ldots, d_{\ell}$. Specifically, we have

$$
\begin{aligned}
\sum_{i=1}^{\ell} \log M_{i} \geq & H\left(W_{\mathcal{L}}\right) \\
= & I\left(X_{0}^{n}, X_{1}^{n}, \ldots, X_{\ell}^{n} ; W_{\mathcal{L}}\right) \\
= & I\left(X_{0}^{n} ; W_{\mathcal{L}}\right)+\sum_{i=1}^{\ell} I\left(X_{i}^{n} ; W_{i} \mid X_{0}^{n}\right) \\
= & h\left(X_{0}^{n}\right)-h\left(X_{0}^{n} \mid W_{\mathcal{L}}\right) \\
& +\sum_{i=1}^{\ell}\left(h\left(X_{i}^{n} \mid X_{0}^{n}\right)-h\left(X_{i}^{n} \mid X_{0}^{n}, W_{i}\right)\right) \\
\geq & \frac{n}{2} \log \frac{\sigma_{0}^{2}}{d_{0}}+\sum_{i=1}^{\ell} \frac{n}{2} \log \frac{\sigma_{i}^{2}}{d_{i}}
\end{aligned}
$$

where (1) follows from the fact that $W_{i}-X_{i}^{n}-X_{0}^{n}-$ $\left(X_{j}^{n}, W_{j}\right)_{j \neq i}$ form a Markov chain for each $i$.

Now we proceed to establish a connection between $d_{0}$ and $\left(d_{1}, d_{2}, \ldots, d_{\ell}\right)$. To this end, we shall examine the following two distortions: $\mathbf{D}_{Y^{n} \mid X_{0}^{n}, W_{\mathcal{L}}}$ and $\tilde{\mathbf{D}}_{Y^{n} \mid X_{0}^{n}, W_{0}^{n}}$, where $W_{0}^{n}=$ $\mathbb{E}\left[X_{0}^{n} \mid W_{\mathcal{L}}\right]$

1) $\mathbf{D}_{Y^{n} \mid X_{0}^{n}, W_{\mathcal{L}}}$ : Observe that

$$
\begin{aligned}
\mathbb{E}\left[Y^{n} \mid X_{0}^{n}, W_{\mathcal{L}}\right] & =\mathbb{E}\left[\sum_{i=1}^{\ell} \frac{\sigma_{N}^{2}}{\sigma_{i}^{2}} X_{i}^{n} \mid X_{0}^{n}, W_{\mathcal{L}}\right] \\
& =\sum_{i=1}^{\ell} \frac{\sigma_{N}^{2}}{\sigma_{i}^{2}} \mathbb{E}\left[X_{i}^{n} \mid X_{0}^{n}, W_{i}\right] .
\end{aligned}
$$

Furthermore, for $i \neq j$

$$
\begin{aligned}
\mathbb{E} & {\left[\left(X_{i}^{n}-\mathbb{E}\left[X_{i}^{n} \mid X_{0}^{n}, W_{i}\right]\right)\left(X_{j}^{n}-\mathbb{E}\left[X_{j}^{n} \mid X_{0}^{n}, W_{j}\right]\right)^{T}\right] } \\
= & \mathbb{E}\left[\left(X_{i}^{n}-\mathbb{E}\left[X_{i}^{n} \mid X_{0}^{n}, X_{j}^{n}, W_{i}, W_{j}\right]\right)\right. \\
& \left.\times\left(X_{j}^{n}-\mathbb{E}\left[X_{j}^{n} \mid X_{0}^{n}, W_{j}\right]\right)^{T}\right] \\
= & 0,
\end{aligned}
$$

where the last equality follows from Lemma 1 . Therefore, we have

$$
\mathbf{D}_{Y^{n} \mid X_{0}^{n}, W_{\mathcal{L}}}=\sum_{i=1}^{\ell}\left(\frac{\sigma_{N}^{2}}{\sigma_{i}^{2}}\right)^{2} d_{i} .
$$

2) $\tilde{\mathbf{D}}_{Y^{n} \mid X_{0}^{n} W_{0}^{n}}$ : Note that

$$
\begin{aligned}
\frac{1}{n} \mathbb{E}\left[\left(X_{0}^{n}-W_{0}^{n}\right)\left(X_{0}^{n}-W_{0}^{n}\right)^{T}\right] & =d_{0}, \\
\frac{1}{n} \mathbb{E}\left[\left(X_{0}^{n}-W_{0}^{n}\right)\left(W_{0}^{n}\right)^{T}\right] & =0,
\end{aligned}
$$


which implies

$$
\frac{1}{n} \mathbb{E}\left[X_{0}^{n}\left(W_{0}^{n}\right)^{T}\right]=\frac{1}{n} \mathbb{E}\left[W_{0}^{n}\left(W_{0}^{n}\right)^{T}\right]=\sigma_{0}^{2}-d_{0} .
$$

Since $N^{n}$ is independent of $W_{0}^{n}$, we have

$$
\frac{1}{n} \mathbb{E}\left[Y^{n}\left(W_{0}^{n}\right)^{T}\right]=\frac{1}{n} \mathbb{E}\left[X_{0}^{n}\left(W_{0}^{n}\right)^{T}\right]=\sigma_{0}^{2}-d_{0} .
$$

Therefore, the reduced covariance matrix of $X_{0}^{n}, Y^{n}$, and $W_{0}^{n}$ (by juxtaposing them into a $3 \times n$ random matrix) is given by

$$
\begin{aligned}
\boldsymbol{\Sigma}_{\left(\left(X_{0}^{n}\right)^{T},\left(Y^{n}\right)^{T},\left(W_{0}^{n}\right)^{T}\right)^{T}} & \\
& =\left(\begin{array}{ccc}
\sigma_{0}^{2} & \sigma_{0}^{2}-\sigma_{N}^{2} & \sigma_{0}^{2}-d_{0} \\
\sigma_{0}^{2}-\sigma_{N}^{2} & \sigma_{0}^{2}-\sigma_{N}^{2} & \sigma_{0}^{2}-d_{0} \\
\sigma_{0}^{2}-d_{0} & \sigma_{0}^{2}-d_{0} & \sigma_{0}^{2}-d_{0}
\end{array}\right) .
\end{aligned}
$$

Now it can be readily verified that

$$
\tilde{\mathbf{D}}_{Y^{n} \mid X_{0}^{n}, W_{0}^{n}}=\sigma_{N}^{2}-\frac{\sigma_{N}^{4}}{d_{0}} .
$$

By Lemma 2 and Lemma 3

$$
\mathbf{D}_{Y^{n} \mid X_{0}^{n}, W_{\mathcal{L}}} \leq \mathbf{D}_{Y^{n} \mid X_{0}^{n}, W_{0}^{n}} \leq \tilde{\mathbf{D}}_{Y^{n} \mid X_{0}^{n}, W_{0}^{n}}
$$

which, in view of (2) and (3), implies that

$$
\frac{1}{d_{0}} \leq \frac{1}{\sigma_{N}^{2}}-\sum_{i=1}^{\ell} \frac{d_{i}}{\sigma_{i}^{4}}
$$

Therefore, we have

$$
R^{\star}(d) \geq \underline{R}^{\star}(d)
$$

where

$$
\begin{aligned}
\underline{R}^{\star}(d) \triangleq \min _{d_{i}, i=0,1, \ldots, \ell} & \frac{1}{2} \log \frac{\sigma_{0}^{2}}{d_{0}}+\sum_{i=1}^{\ell} \frac{1}{2} \log \frac{\sigma_{i}^{2}}{d_{i}} \\
\text { subject to } & \frac{1}{d_{0}} \leq \frac{1}{\sigma_{N}^{2}}-\sum_{i=1}^{\ell} \frac{d_{i}}{\sigma_{i}^{4}} \\
& \sigma_{N}^{2}<d_{0} \leq d \\
& 0<d_{i} \leq \sigma_{i}^{2}, \quad i=1,2, \ldots, \ell .
\end{aligned}
$$

\section{B. Upper Bound}

We shall proceed to derive a matching upper bound on $R^{\star}(d)$. Let $U_{1}, U_{2}, \ldots, U_{\ell}$ be $\ell$ auxiliary random variables jointly distributed with the generic source variables $X_{0}, X_{1}, \ldots, X_{\ell}$ such that such that $U_{i}-X_{i}-\left(X_{0}, X_{j}, U_{j}, j \neq i\right)$ form a Markov chain for $i=1,2, \ldots, \ell$. The well-known Berger-Tung upper bound [1], [20] states that

$$
R^{\star}(d) \leq I\left(X_{1}, X_{2}, \ldots, X_{\ell} ; U_{\mathcal{L}}\right)
$$

if $\mathbf{D}_{X_{0} \mid U_{\mathcal{L}}} \leq d$, where $U_{\mathcal{L}}=\left(U_{1}, U_{2}, \ldots, U_{\ell}\right)$. Now let $U_{i}=$ $c_{i} X_{i}+Z_{i}, \bar{i}=1,2, \ldots, \ell$, where $c_{i}$ is a constant and $Z_{i}$ is a zero-mean unit-variance Gaussian random variable independent of everything else. With a slight abuse of notation, we define $d_{0}=\mathbf{D}_{X_{0} \mid U_{\mathcal{L}}}$ and $d_{i}=\mathbf{D}_{X_{i} \mid X_{0}, U_{i}}, i=1,2, \ldots, \ell$. It is clear that $d_{i}$ can take any value in $\left(0, \sigma_{i}^{2}\right], i=1,2, \ldots, \ell$. In view of the derivation that leads to (2) and (3), we must have

$$
\begin{aligned}
& \mathbf{D}_{Y \mid X_{0}, U_{\mathcal{L}}}=\sum_{i=1}^{\ell}\left(\frac{\sigma_{N}^{2}}{\sigma_{i}^{2}}\right)^{2} d_{i} \\
& \tilde{\mathbf{D}}_{Y \mid X_{0}, U_{0}}=\sigma_{N}^{2}-\frac{\sigma_{N}^{4}}{d_{0}},
\end{aligned}
$$

where $U_{0}=\mathbb{E}\left[X_{0} \mid U_{\mathcal{L}}\right]$. By invoking the fact that $U_{0}$ is a sufficient statistic of $U_{\mathcal{L}}$ for estimating $\left(X_{0}, Y\right)$ and the fact that linear MMSE estimation coincides with MMSE estimation for joint Gaussian distributions, it can be shown that

$$
\mathbf{D}_{Y \mid X_{0}, U_{\mathcal{L}}}=\mathbf{D}_{Y \mid X_{0}, U_{0}}=\tilde{\mathbf{D}}_{Y \mid X_{0}, U_{0}}
$$

Therefore, we have

$$
\frac{1}{d_{0}}=\frac{1}{\sigma_{N}^{2}}-\sum_{i=1}^{\ell} \frac{d_{i}}{\sigma_{i}^{4}},
$$

which implies $d_{0}>\sigma_{N}^{2}$ if we restrict $d_{i}$ in $\left(0, \sigma_{i}^{2}\right]$, $i=1,2, \ldots, \ell$.

Note that

$$
\begin{aligned}
I\left(X_{1}, X_{2}, \ldots, X_{\ell} ; U_{\mathcal{L}}\right) & =I\left(X_{0}, X_{1}, X_{2}, \ldots, X_{\ell} ; U_{\mathcal{L}}\right) \\
= & I\left(X_{0} ; U_{\mathcal{L}}\right)+\sum_{i=1}^{\ell} I\left(X_{i} ; U_{i} \mid X_{0}\right) \\
= & h\left(X_{0}\right)-h\left(X_{0} \mid U_{\mathcal{L}}\right) \\
\quad & +\sum_{i=1}^{\ell}\left(h\left(X_{i} \mid X_{0}\right)-h\left(X_{i} \mid X_{0}, U_{i}\right)\right) \\
= & \frac{1}{2} \log \frac{\sigma_{0}^{2}}{d_{0}}+\sum_{i=1}^{\ell} \frac{1}{2} \log \frac{\sigma_{i}^{2}}{d_{i}} .
\end{aligned}
$$

Therefore, we have

$$
R^{\star}(d) \leq \bar{R}^{\star}(d)
$$

where

$$
\begin{aligned}
\bar{R}^{\star}(d) \triangleq \min _{d_{i}, i=0,1, \ldots, \ell} & \frac{1}{2} \log \frac{\sigma_{0}^{2}}{d_{0}}+\sum_{i=1}^{\ell} \frac{1}{2} \log \frac{\sigma_{i}^{2}}{d_{i}} \quad\left(\mathbf{P}_{1}^{\prime}\right) \\
\text { subject to } \quad & \frac{1}{d_{0}}=\frac{1}{\sigma_{N}^{2}}-\sum_{i=1}^{\ell} \frac{d_{i}}{\sigma_{i}^{4}} \\
& \sigma_{N}^{2}<d_{0} \leq d \\
& 0<d_{i} \leq \sigma_{i}^{2}, \quad i=1,2, \ldots, \ell .
\end{aligned}
$$

It can be seen that the minimization problem associated with $\underline{R}^{\star}(d)$ is almost identical with the one associated with $\bar{R}^{\star}(d)$ except that " $\leq$ " in (4) is replaced by "=" in (5). Since $\frac{1}{2} \log \frac{\sigma_{0}^{2}}{d_{0}}$ 
is a strictly monotone decreasing function of $d_{0}$, it follows that any optimal solution $\left(d_{0}^{*}, d_{1}^{*}, \ldots, d_{l}^{*}\right)$ to $\left(\mathbf{P}_{1}\right)$ must satisfy

$$
\frac{1}{d_{0}^{*}}=\frac{1}{\sigma_{N}^{2}}-\sum_{i=1}^{\ell} \frac{d_{i}^{*}}{\sigma_{i}^{4}} .
$$

Therefore, $\left(\mathbf{P}_{1}\right)$ and $\left(\mathbf{P}_{1}^{\prime}\right)$ are equivalent. As a consequence, we have

$$
R^{\star}(d)=\underline{R}^{\star}(d)=\bar{R}^{\star}(d) .
$$

\section{The Gaussian Multiterminal Source CODING PROBLEM}

Now we shall apply the new method to the Gaussian multiterminal source coding problem, which is still largely an open problem. Let $\left(X_{1}, X_{2}, \ldots, X_{\ell}\right)^{T}$ be a Gaussian source with mean zero and positive definite covariance matrix $\boldsymbol{\Sigma}_{\mathbf{X}}$. For the reason which will be clear later, we shall augment the probability space by introducing additional random variables. Let $\mathbb{N}\left(\boldsymbol{\Sigma}_{\mathbf{X}}\right)$ be the set of positive definite covariance matrices $\boldsymbol{\Sigma}_{\mathbf{N}}$ such that $\boldsymbol{\Sigma}_{\mathbf{X}}^{-1}+\boldsymbol{\Sigma}_{\mathbf{N}}^{-1}$ is a diagonal matrix. Note that $\mathbb{N}\left(\boldsymbol{\Sigma}_{\mathbf{X}}\right)$ is nonempty since $\left(\boldsymbol{\Delta}-\boldsymbol{\Sigma}_{\mathbf{X}}^{-1}\right)^{-1} \in \mathbb{N}\left(\boldsymbol{\Sigma}_{\mathbf{X}}\right)$ for any positive definite diagonal matrix $\boldsymbol{\Delta}$ with sufficiently large diagonal entries. Let $\left(N_{1}, N_{2}, \ldots, N_{\ell}\right)^{T}$ be a Gaussian random vector with mean zero and covariance matrix $\boldsymbol{\Sigma}_{\mathbf{N}} \in \mathbb{N}\left(\boldsymbol{\Sigma}_{\mathbf{X}}\right)$. We assume $\left(N_{1}, N_{2}, \ldots, N_{\ell}\right)^{T}$ is independent of $\left(X_{1}, X_{2}, \ldots, X_{\ell}\right)^{T}$ and define $\left(Y_{1}, Y_{2}, \ldots, Y_{\ell}\right)^{T}=\left(X_{1}+N_{1}, X_{2}+N_{2}, \ldots, X_{\ell}+N_{\ell}\right)^{T}$. Note that $X_{1}, X_{2}, \ldots, X_{\ell}$ are independent conditioned on $\left(Y_{1}, Y_{2}, \ldots, Y_{\ell}\right)^{T}$. This follows from the fact that $\left(X_{1}, X_{2}, \ldots, X_{\ell}\right)^{T}$ is jointly Gaussian with $\left(Y_{1}, Y_{2}, \ldots, Y_{\ell}\right)^{T}$ and the fact that the distortion covariance matrix of the linear MMSE estimation for $\left(X_{1}, X_{2}, \ldots, X_{\ell}\right)^{T}$ given $\left(Y_{1}, Y_{2}, \ldots, Y_{\ell}\right)^{T}$ is $\left(\boldsymbol{\Sigma}_{\mathbf{X}}^{-1}+\boldsymbol{\Sigma}_{\mathbf{N}}^{-1}\right)^{-1}$, which is a diagonal matrix. Intuitively, one may view $\left(Y_{1}, Y_{2}, \ldots, Y_{\ell}\right)^{T}$ as a remote source. However, it should be noted that due to its vector nature, $\left(Y_{1}, Y_{2}, \ldots, Y_{\ell}\right)^{T}$ is different from the remote source in the standard scalar Gaussian CEO problem.

Let $\quad\left\{\left(\left(X_{1}(t), X_{2}(t), \ldots, X_{\ell}(t)\right)^{T},\left(Y_{1}(t), Y_{2}(t), \ldots\right.\right.\right.$, $\left.\left.\left.Y_{\ell}(t)\right)^{T},\left(N_{1}(t), N_{2}(t), \ldots, N_{\ell}(t)\right)^{T}\right)\right\}_{t=1}^{\infty}$ be i.i.d. copies of $\left(\left(X_{1}, X_{2}, \ldots, X_{\ell}\right)^{T},\left(Y_{1}, Y_{2}, \ldots, Y_{\ell}\right)^{T},\left(N_{1}, N_{2}, \ldots, N_{\ell}\right)^{T}\right)$.

Definition 2: We say sum rate $R$ is achievable with respect to distortion constraint $\mathbf{d}=\left(d_{1}, d_{2}, \ldots, d_{\ell}\right)^{T}$ if there exist $\ell$ encoding functions $f_{i}: \mathbb{R}^{n} \rightarrow\left\{1,2, \ldots, M_{i}\right\}, i=1,2, \ldots, \ell$, such that

$$
\begin{aligned}
\frac{1}{n} \sum_{i=1}^{\ell} \log M_{i} & \leq R \\
\mathbf{D}_{X_{i}^{n} \mid W_{\mathcal{L}}} & \leq d_{i}, \quad i=1,2, \ldots, \ell,
\end{aligned}
$$

where $W_{\mathcal{L}}=\left(W_{1}, W_{2}, \ldots, W_{\ell}\right)^{T}$ with $W_{i}=f_{i}\left(X_{i}^{n}\right), i=$ $1,2, \ldots, \ell$. The minimum sum rate $R^{\star}(\mathbf{d})$ is the infimum of all achievable sum rates with respect to distortion constraint $\mathbf{d}$.

Remark: It is clear that we can restrict our attention to the distortion constraints satisfying $d_{i} \in\left(0, \sigma_{X_{i}}^{2}\right], i=1,2, \ldots, \ell$, where $\sigma_{X_{i}}^{2}$ is the $i$ th diagonal entry of $\boldsymbol{\Sigma}_{\mathbf{X}}$.

\section{A. Lower Bound}

Let $f_{i}: \mathbb{R}^{n} \rightarrow\left\{1,2, \ldots, M_{i}\right\}, i=1,2, \ldots, \ell$, be $\ell$ encoding functions such that $\mathbf{D}_{X_{i}^{n} \mid W_{\mathcal{L}}} \leq d_{i}, i=1,2, \ldots, \ell$. We shall denote $\left(\left(X_{1}^{n}\right)^{T},\left(X_{2}^{n}\right)^{T}, \ldots,\left(X_{\ell}^{n}\right)^{T}\right)^{T}$, $\left(\left(Y_{1}^{n}\right)^{T},\left(Y_{2}^{n}\right)^{T}, \ldots,\left(Y_{\ell}^{n}\right)^{T}\right)^{T}$, $\left(\left(N_{1}^{n}\right)^{T},\left(N_{2}^{n}\right)^{T}, \ldots,\left(N_{\ell}^{n}\right)^{T}\right)^{T}$ by $\mathbf{X}, \mathbf{Y}$, and $\mathbf{N}$, respectively. Define $\sigma_{i}^{2}=\mathbf{D}_{X_{i}^{n} \mid \mathbf{Y}}, i=1,2, \ldots, \ell$. It is easy to see that $\sigma_{1}^{2}, \sigma_{2}^{2}, \ldots, \sigma_{\ell}^{2}$ are the diagonal entries of $\left(\boldsymbol{\Sigma}_{\mathbf{X}}^{-1}+\boldsymbol{\Sigma}_{\mathbf{N}}^{-1}\right)^{-1}$. Moreover, define $\mathbf{D}=\mathbf{D}_{\mathbf{X} \mid W_{\mathcal{L}}}$ and $\gamma_{i}=\mathbf{D}_{X_{i}^{n} \mid \mathbf{Y}, W_{i}}$, $i=1,2, \ldots, \ell$. Note that $\mathbf{D}$ and $\gamma_{i}, i=1,2, \ldots, \ell$, satisfy $\mathbf{0} \prec \mathbf{D} \preceq \boldsymbol{\Sigma}_{\mathbf{X}}, d_{i, i} \leq d_{i}$, and $\gamma_{i} \in\left(0, \sigma_{i}^{2}\right], i=1,2, \ldots, \ell$, where $d_{i, i}$ is the $i$ th diagonal entry of $\mathbf{D}$.

First we shall derive a lower bound on the sum rate and express it as a function of $\mathbf{D}, \gamma_{1}, \ldots, \gamma_{\ell}$. Specifically, we have

$$
\begin{aligned}
\sum_{i=1}^{\ell} \log M_{i} \geq & H\left(W_{\mathcal{L}}\right) \\
= & I\left(\mathbf{X}, \mathbf{Y} ; W_{\mathcal{L}}\right) \\
= & I\left(\mathbf{Y} ; W_{\mathcal{L}}\right)+\sum_{i=1}^{\ell} I\left(X_{i}^{n} ; W_{i} \mid \mathbf{Y}\right) \\
= & h(\mathbf{Y})-h\left(\mathbf{Y} \mid W_{\mathcal{L}}\right) \\
& +\sum_{i=1}^{\ell}\left(h\left(X_{i}^{n} \mid \mathbf{Y}\right)-h\left(X_{i}^{n} \mid \mathbf{Y}, W_{i}\right)\right) \\
\geq & \frac{n}{2} \log \frac{\left|\boldsymbol{\Sigma}_{\mathbf{X}}+\mathbf{\Sigma}_{\mathbf{N}}\right|}{\left|\mathbf{D}+\mathbf{\Sigma}_{\mathbf{N}}\right|}+\frac{n}{2} \sum_{i=1}^{\ell} \log \frac{\sigma_{i}^{2}}{\gamma_{i}}
\end{aligned}
$$

where (7) follows from the fact that $W_{i}-X_{i}^{n}-\mathbf{Y}-$ $\left(X_{j}^{n}, W_{j}\right)_{j \neq i}$ form a Markov chain for each $i$.

Now we proceed to establish a connection between $\mathbf{D}$ and $\left(\gamma_{1}, \gamma_{2}, \ldots, \gamma_{\ell}\right)$. To this end, we shall examine the following

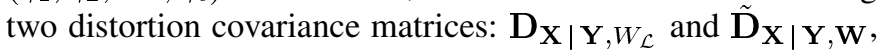
where $\mathbf{W}=\mathbb{E}\left[\mathbf{X} \mid W_{\mathcal{L}}\right]$.

1) $\mathbf{D}_{\mathbf{X} \mid \mathbf{Y}, W_{\mathcal{L}}}$ : Note that

$$
\mathbf{D}_{X_{i}^{n} \mid \mathbf{Y}, W_{\mathcal{L}}}=\mathbf{D}_{X_{i}^{n} \mid \mathbf{Y}, W_{i}}=\gamma_{i}, \quad i=1,2, \ldots, \ell .
$$

Moreover, for $i \neq j$

$$
\begin{aligned}
\mathbb{E}[ & \left.\left(X_{i}^{n}-\mathbb{E}\left[X_{i}^{n} \mid \mathbf{Y}, W_{\mathcal{L}}\right]\right)\left(X_{j}^{n}-\mathbb{E}\left[X_{j}^{n} \mid \mathbf{Y}, W_{\mathcal{L}}\right]\right)^{T}\right] \\
= & \mathbb{E}\left[\left(X_{i}^{n}-\mathbb{E}\left[X_{i}^{n} \mid \mathbf{Y}, W_{i}\right]\right)\right. \\
& \left.\times\left(X_{j}^{n}-\mathbb{E}\left[X_{j}^{n} \mid \mathbf{Y}, W_{j}\right]\right)^{T}\right] \\
= & \mathbb{E}\left[\left(X_{i}^{n}-\mathbb{E}\left[X_{i}^{n} \mid \mathbf{Y}, X_{j}, W_{i}, W_{j}\right]\right)\right. \\
& \left.\times\left(X_{j}^{n}-\mathbb{E}\left[X_{j}^{n} \mid \mathbf{Y}, W_{j}\right]\right)^{T}\right] \\
= & 0,
\end{aligned}
$$

where the last equality follows from Lemma 1 . Therefore, we have

$\mathbf{D}_{\mathbf{X} \mid \mathbf{Y}, W_{\mathcal{L}}}=\left(\begin{array}{cccc}\gamma_{1} & 0 & \cdots & 0 \\ 0 & \gamma_{2} & \cdots & 0 \\ \vdots & \vdots & \ddots & \vdots \\ 0 & 0 & \cdots & \gamma_{\ell}\end{array}\right) \triangleq \operatorname{diag}\left(\gamma_{1}, \gamma_{2}, \ldots, \gamma_{\ell}\right)$. 
2) $\tilde{\mathbf{D}}_{\mathbf{X} \mid \mathbf{Y}, \mathbf{W}}$ : Note that

$$
\begin{aligned}
\frac{1}{n} \mathbb{E}\left[(\mathbf{X}-\mathbf{W})(\mathbf{X}-\mathbf{W})^{T}\right] & =\mathbf{D}, \\
\frac{1}{n} \mathbb{E}\left[(\mathbf{X}-\mathbf{W}) \mathbf{W}^{T}\right] & =\mathbf{0},
\end{aligned}
$$

which implies

$$
\frac{1}{n} \mathbb{E}\left[\mathbf{X W}^{T}\right]=\frac{1}{n} \mathbb{E}\left[\mathbf{W} \mathbf{W}^{T}\right]=\boldsymbol{\Sigma}_{\mathbf{X}}-\mathbf{D} .
$$

Since $\mathbf{N}$ is independent of $\mathbf{W}$, we have

$$
\frac{1}{n} \mathbb{E}\left[\mathbf{Y W} \mathbf{W}^{T}\right]=\frac{1}{n} \mathbb{E}\left[\mathbf{X W}^{T}\right]=\mathbf{\Sigma}_{\mathbf{X}}-\mathbf{D} .
$$

Therefore, the reduced covariance matrix of $\mathbf{X}, \mathbf{Y}$, and $\mathbf{W}$ (by juxtaposing them into a $3 \ell \times n$ random matrix) is given by

$$
\boldsymbol{\Sigma}_{\left(\mathbf{X}^{T}, \mathbf{Y}^{T}, \mathbf{W}^{T}\right)^{T}}=\left(\begin{array}{ccc}
\boldsymbol{\Sigma}_{\mathbf{X}} & \boldsymbol{\Sigma}_{\mathbf{X}} & \boldsymbol{\Sigma}_{\mathbf{X}}-\mathbf{D} \\
\boldsymbol{\Sigma}_{\mathbf{X}} & \boldsymbol{\Sigma}_{\mathbf{X}}+\boldsymbol{\Sigma}_{\mathbf{N}} & \boldsymbol{\Sigma}_{\mathbf{X}}-\mathbf{D} \\
\boldsymbol{\Sigma}_{\mathbf{X}}-\mathbf{D} & \boldsymbol{\Sigma}_{\mathbf{X}}-\mathbf{D} & \boldsymbol{\Sigma}_{\mathbf{X}}-\mathbf{D}
\end{array}\right) .
$$

Now it can be readily verified that

$$
\tilde{\mathbf{D}}_{\mathbf{X} \mid \mathbf{Y}, \mathbf{W}}=\left(\mathbf{D}^{-1}+\boldsymbol{\Sigma}_{\mathbf{N}}^{-1}\right)^{-1} .
$$

By Lemma 2 and Lemma 3, we have

$$
\mathbf{D}_{\mathbf{X} \mid \mathbf{Y}, W_{\mathcal{L}}} \preceq \mathbf{D}_{\mathbf{X} \mid \mathbf{Y}, \mathbf{W}} \preceq \tilde{\mathbf{D}}_{\mathbf{X} \mid \mathbf{Y}, \mathbf{W}}
$$

which, in view of (8) and (9), implies that

$$
\operatorname{diag}\left(\gamma_{1}, \gamma_{2}, \ldots, \gamma_{\ell}\right) \preceq\left(\mathbf{D}^{-1}+\boldsymbol{\Sigma}_{\mathbf{N}}^{-1}\right)^{-1} .
$$

Therefore, we have obtained the following lower bound.

Theorem 1: For any $\boldsymbol{\Sigma}_{\mathbf{N}} \in \mathbb{N}\left(\boldsymbol{\Sigma}_{\mathbf{X}}\right)$, we have $R^{\star}(\mathbf{d}) \geq$ $\underline{R}^{\star}\left(\mathrm{d}, \Sigma_{\mathrm{N}}\right)$, where

$$
\begin{aligned}
\underline{R}^{\star}\left(\mathbf{d}, \boldsymbol{\Sigma}_{\mathbf{N}}\right) \triangleq \min _{\mathbf{D}, \gamma_{i}, i=1,2, \ldots, \ell} & \frac{1}{2} \log \frac{\left|\boldsymbol{\Sigma}_{\mathbf{X}}+\mathbf{\Sigma}_{\mathbf{N}}\right|}{\left|\mathbf{D}+\mathbf{\Sigma}_{\mathbf{N}}\right|} \\
& +\frac{1}{2} \sum_{i=1}^{\ell} \log \frac{\sigma_{i}^{2}}{\gamma_{i}} \quad\left(\mathbf{P}_{2}\right) \\
\text { subject to } & \operatorname{diag}\left(\gamma_{1}, \gamma_{2}, \ldots, \gamma_{\ell}\right) \\
& \preceq\left(\mathbf{D}^{-1}+\boldsymbol{\Sigma}_{\mathbf{N}}^{-1}\right)^{-1} \\
& \mathbf{0} \prec \mathbf{D} \preceq \boldsymbol{\Sigma}_{\mathbf{X}} \\
& d_{i, i} \leq d_{i}, \quad i=1,2, \ldots, \ell \\
& 0<\gamma_{i} \leq \sigma_{i}^{2}, \quad i=1,2, \ldots, \ell .
\end{aligned}
$$

\section{B. Upper Bound}

Now we proceed to derive an upper bound on $R^{\star}(\mathbf{d})$. We shall denote $\left(X_{1}, \ldots, X_{\ell}\right)^{T}$ and $\left(Y_{1}, \ldots, Y_{\ell}\right)^{T}$ by $X_{\mathcal{L}}$ and $Y_{\mathcal{L}}$, respectively. Let $U_{1}, U_{2}, \ldots, U_{\ell}$ be $\ell$ auxiliary random variables jointly distributed with $X_{\mathcal{L}}$ and $Y_{\mathcal{L}}$ such that such that $U_{i}-X_{i}-\left(Y_{\mathcal{L}}, X_{j}, U_{j}, j \neq i\right)$ form a Markov chain for
(8) $i=1,2, \ldots, \ell$. For the Gaussian multiterminal source coding problem, the Berger-Tung upper bound [1], [20] states that

$$
R^{\star}(\mathbf{d}) \leq I\left(X_{\mathcal{L}} ; U_{\mathcal{L}}\right)
$$

if $\mathbf{D}_{X_{i} \mid U_{\mathcal{L}}} \leq d_{i}, i=1,2, \ldots, \ell$, where $U_{\mathcal{L}}=$ $\left(U_{1}, U_{2}, \ldots, U_{\ell}\right)^{T}$. Now let $U_{\mathcal{L}}$ be generated by $X_{\mathcal{L}}$ through parallelled Gaussian test channels, i.e., $U_{i}=c_{i} X_{i}+Z_{i}$, $i=1,2, \ldots, \ell$, where $c_{i} \in[0, \infty)$ is a constant and $Z_{i}$ is a zero-mean unit-variance Gaussian random variable independent of everything else. With a slight abuse of notation, we define $\mathbf{D}=\mathbf{D}_{X_{\mathcal{L}} \mid U_{\mathcal{L}}}$ and $\gamma_{i}=\mathbf{D}_{X_{i} \mid Y_{\mathcal{L}}, U_{i}}$, $i=1,2, \ldots, \ell$. It is clear that $\gamma_{i}$ can take any value in $\left(0, \sigma_{i}^{2}\right]$, $i=1,2, \ldots, \ell$. Moreover, $\mathbf{D}_{X_{i} \mid U_{\mathcal{S}}} \leq d_{i}$ is equivalent to $d_{i, i} \leq d_{i}, i=1,2, \ldots, \ell$. In view of the derivation that leads to (8) and (9), we must have

$$
\begin{aligned}
& \mathbf{D}_{X_{\mathcal{L}} \mid Y_{\mathcal{L}}, U_{\mathcal{L}}}=\operatorname{diag}\left(\gamma_{1}, \gamma_{2}, \ldots, \gamma_{l}\right) \\
& \tilde{\mathbf{D}}_{X_{\mathcal{L}} \mid Y_{\mathcal{L}}, \hat{U}_{\mathcal{L}}}=\left(\mathbf{D}^{-1}+\boldsymbol{\Sigma}_{\mathbf{N}}^{-1}\right)^{-1}
\end{aligned}
$$

where $\hat{U}_{\mathcal{L}}=\mathbb{E}\left[X_{\mathcal{L}} \mid U_{\mathcal{L}}\right]$.

By invoking the fact that $\hat{U}_{\mathcal{L}}$ is a sufficient statistic of $U_{\mathcal{L}}$ for estimating $\left(X_{\mathcal{L}}, Y_{\mathcal{L}}\right)$ and the fact that linear MMSE estimation coincides with MMSE estimation for joint Gaussian distributions, it can be readily shown that

$$
\mathbf{D}_{X_{\mathcal{L}} \mid Y_{\mathcal{L}}, U_{\mathcal{L}}}=\mathbf{D}_{X_{\mathcal{L}} \mid Y_{\mathcal{L}}, \hat{U}_{\mathcal{L}}}=\tilde{\mathbf{D}}_{X_{\mathcal{L}} \mid Y_{\mathcal{L}}, \hat{U}_{\mathcal{L}}} .
$$

Therefore, we have

$$
\operatorname{diag}\left(\gamma_{1}, \gamma_{2}, \ldots, \gamma_{\ell}\right)=\left(\mathbf{D}^{-1}+\boldsymbol{\Sigma}_{\mathbf{N}}^{-1}\right)^{-1} .
$$

It is clear that $\mathbf{D}$ must satisfy $\mathbf{0} \prec \mathbf{D} \preceq \boldsymbol{\Sigma}_{\mathbf{X}}$ if we restrict $\gamma_{i}$ in $\left(0, \sigma_{i}^{2}\right], i=1,2, \ldots, \ell$.

Note that

$$
\begin{aligned}
I\left(X_{\mathcal{L}} ; U_{\mathcal{L}}\right)= & I\left(Y_{\mathcal{L}}, X_{\mathcal{L}} ; U_{\mathcal{L}}\right) \\
= & I\left(Y_{\mathcal{L}} ; U_{\mathcal{L}}\right)+\sum_{i=1}^{\ell} I\left(X_{i} ; U_{i} \mid Y_{\mathcal{L}}\right) \\
= & h\left(Y_{\mathcal{L}}\right)-h\left(Y_{\mathcal{L}} \mid U_{\mathcal{L}}\right) \\
& +\sum_{i=1}^{\ell}\left(h\left(X_{i} \mid Y_{\mathcal{L}}\right)-h\left(X_{i} \mid Y_{\mathcal{L}}, U_{i}\right)\right) \\
= & \frac{1}{2} \log \frac{\left|\Sigma_{\mathbf{X}}+\Sigma_{\mathbf{N}}\right|}{\left|\mathbf{D}+\Sigma_{\mathbf{N}}\right|}+\frac{1}{2} \sum_{i=1}^{\ell} \log \frac{\sigma_{i}^{2}}{\gamma_{i}} .
\end{aligned}
$$

Therefore, we have obtained the following upper bound.

Theorem 2: For any $\boldsymbol{\Sigma}_{\mathbf{N}} \in \mathbb{N}\left(\boldsymbol{\Sigma}_{\mathbf{X}}\right)$, we have $R^{\star}(\mathbf{d}) \leq$ $\bar{R}^{\star}\left(\mathbf{d}, \boldsymbol{\Sigma}_{\mathbf{N}}\right)$, where

$$
\begin{aligned}
\bar{R}^{\star}\left(\mathbf{d}, \boldsymbol{\Sigma}_{\mathbf{N}}\right) \triangleq \min _{\mathbf{D}, \gamma_{i}, i=1,2, \ldots, \ell} \quad \frac{1}{2} \log \frac{\left|\boldsymbol{\Sigma}_{\mathbf{X}}+\boldsymbol{\Sigma}_{\mathbf{N}}\right|}{\left|\mathbf{D}+\boldsymbol{\Sigma}_{\mathbf{N}}\right|} \\
+\frac{1}{2} \sum_{i=1}^{\ell} \log \frac{\sigma_{i}^{2}}{\gamma_{i}}
\end{aligned}
$$




$$
\begin{aligned}
& \text { subject to } \operatorname{diag}\left(\gamma_{1}, \gamma_{2}, \ldots, \gamma_{\ell}\right) \\
& =\left(\mathbf{D}^{-1}+\mathbf{\Sigma}_{\mathbf{N}}^{-1}\right)^{-1} \\
& \mathbf{0} \prec \mathbf{D} \preceq \boldsymbol{\Sigma}_{\mathbf{X}} \\
& d_{i, i} \leq d_{i}, \quad i=1,2, \ldots, \ell \\
& 0<\gamma_{i} \leq \sigma_{i}^{2}, \quad i=1,2, \ldots, \ell \text {. }
\end{aligned}
$$

Remark: It is worth noting that $\bar{R}^{\star}\left(\mathbf{d}, \boldsymbol{\Sigma}_{\mathbf{N}}\right)$ does not depend on $\boldsymbol{\Sigma}_{\mathbf{N}}$ if we restrict $\boldsymbol{\Sigma}_{\mathbf{N}}$ in $\mathbb{N}\left(\boldsymbol{\Sigma}_{\mathbf{X}}\right)$. This is because

$$
I\left(X_{\mathcal{L}} ; U_{\mathcal{L}}\right)=h\left(X_{\mathcal{L}}\right)-h\left(X_{\mathcal{L}} \mid U_{\mathcal{L}}\right)=\frac{1}{2} \log \frac{\left|\boldsymbol{\Sigma}_{\mathbf{X}}\right|}{|\mathbf{D}|}
$$

and the construction of $U_{\mathcal{L}}$ implies that $\mathbf{D}$ can be any positive definite matrix with the properties that $\mathbf{0} \prec \mathbf{D} \preceq \boldsymbol{\Sigma}_{\mathbf{X}}, d_{i, i} \leq d_{i}$, and $\mathbf{D}^{-1}-\boldsymbol{\Sigma}_{\mathbf{X}}^{-1}$ is a positive semidefinite diagonal matrix; as a consequence, we can write $\bar{R}^{\star}\left(\mathbf{d}, \boldsymbol{\Sigma}_{\mathbf{N}}\right)$ alternatively as

$$
\begin{aligned}
\bar{R}^{\star}\left(\mathbf{d}, \boldsymbol{\Sigma}_{\mathbf{N}}\right)=\min _{\mathbf{D}} \frac{1}{2} \log \frac{\left|\boldsymbol{\Sigma}_{\mathbf{X}}\right|}{|\mathbf{D}|} \\
\text { subject to } \quad \mathbf{0} \prec \mathbf{D} \preceq \boldsymbol{\Sigma}_{\mathbf{X}} \\
d_{i, i} \leq d_{i}, \quad i=1,2, \ldots, \ell \\
\mathbf{D} \in \mathbb{D}\left(\boldsymbol{\Sigma}_{\mathbf{X}}\right),
\end{aligned}
$$

where $\mathbb{D}\left(\boldsymbol{\Sigma}_{\mathbf{X}}\right)$ is the set of positive definite matrices $\mathbf{D}$ such that $\mathbf{D}^{-1}-\Sigma_{\mathbf{X}}^{-1}$ is a positive semidefinite diagonal matrix.

It can be seen that $\left(\mathbf{P}_{2}\right)$ and $\left(\mathbf{P}_{2}^{\prime}\right)$ are almost identical except that " $\preceq$ " in (10) is replaced by "=" in (11). Therefore, we immediately obtain the following result.

Corollary 1: If there exists $\boldsymbol{\Sigma}_{\mathbf{N}} \in \mathbb{N}\left(\boldsymbol{\Sigma}_{\mathbf{X}}\right)$ such that an optimal solution $\left(\mathbf{D}, \gamma_{1}, \gamma_{2}, \ldots, \gamma_{\ell}\right)$ to $\left(\mathbf{P}_{2}\right)$ satisfies

$$
\operatorname{diag}\left(\gamma_{1}, \gamma_{2}, \ldots, \gamma_{\ell}\right)=\left(\mathbf{D}^{-1}+\boldsymbol{\Sigma}_{\mathbf{N}}^{-1}\right)^{-1}
$$

then we have

$$
R^{\star}(\mathbf{d})=\underline{R}^{\star}\left(\mathbf{d}, \boldsymbol{\Sigma}_{\mathbf{N}}\right)=\bar{R}^{\star}\left(\mathbf{d}, \boldsymbol{\Sigma}_{\mathbf{N}}\right)=\frac{1}{2} \log \frac{\left|\boldsymbol{\Sigma}_{\mathbf{X}}\right|}{|\mathbf{D}|} .
$$

\section{On the Tightness of the Lower and Upper Bounds}

In the sequel we shall establish a set of sufficient conditions under which (12) in Corollary 1 can be verified analytically.

Define $\mathbb{D}\left(\mathbf{d}, \boldsymbol{\Sigma}_{\mathbf{X}}\right)=\left\{\mathbf{D} \in \mathbb{D}\left(\boldsymbol{\Sigma}_{\mathbf{X}}\right): d_{i, i}=d_{i}, i=\right.$ $1,2, \ldots, \ell\}$. Note that $\mathbb{D}\left(\mathbf{d}, \boldsymbol{\Sigma}_{\mathbf{X}}\right)$ is nonempty if and only if $d_{i}=\mathbf{D}_{X_{i} \mid U_{\mathcal{L}}}, i=1,2, \ldots, \ell$, for some $U_{\mathcal{L}}$ generated by $X_{\mathcal{L}}$ through parallelled Gaussian test channels. Although it is easy to compute the resulting distortions given the parallelled Gaussian test channels, the reverse direction turns out to be much more difficult. As a consequence, it is a nontrivial task to verify whether $\mathbb{D}\left(\mathbf{d}, \boldsymbol{\Sigma}_{\mathbf{X}}\right)$ is empty or not for a given $\mathbf{d}$. Fortunately, the following result indicates that $\mathbb{D}\left(\mathbf{d}, \boldsymbol{\Sigma}_{\mathbf{X}}\right)$ is always nonempty in the high-resolution regime (i.e., when the entries of $\mathbf{d}$ are sufficiently small).

Theorem 3: The set $\mathbb{D}\left(\mathbf{d}, \boldsymbol{\Sigma}_{\mathbf{X}}\right)$ is nonempty if $d_{i} \leq$ $\mathbf{D}_{X_{i} \mid\left(X_{j}\right)_{j \neq i}}, i=1,2, \ldots, \ell$.

Proof: See Appendix A.
Theorem 3: The set $\mathbb{D}\left(\mathbf{d}, \boldsymbol{\Sigma}_{\mathbf{X}}\right)$ is a singleton if it is nonempty.

Proof: See Appendix B.

Lemma 4: If for some $\mathbf{D} \in \mathbb{D}\left(\mathbf{d}, \boldsymbol{\Sigma}_{\mathbf{X}}\right)$ and $\boldsymbol{\Sigma}_{\mathbf{N}} \in \mathbb{N}\left(\boldsymbol{\Sigma}_{\mathbf{X}}\right)$, there exists a diagonal matrix $\boldsymbol{\Pi}=\operatorname{diag}\left(\pi_{1}, \pi_{2}, \ldots, \pi_{\ell}\right)$ such that $\mathbf{D}\left(\boldsymbol{\Pi}-\left(\mathbf{D}+\boldsymbol{\Sigma}_{\mathbf{N}}\right)^{-1}\right) \mathbf{D}$ is a positive semidefinite matrix with its diagonal entries the same as those of $\left(\mathbf{D}^{-1}+\boldsymbol{\Sigma}_{\mathbf{N}}^{-1}\right)^{-1}$, then we have

$$
R^{\star}(\mathbf{d})=\underline{R}^{\star}\left(\mathbf{d}, \boldsymbol{\Sigma}_{\mathbf{N}}\right)=\bar{R}^{\star}\left(\mathbf{d}, \boldsymbol{\Sigma}_{\mathbf{N}}\right)=\frac{1}{2} \log \frac{\left|\boldsymbol{\Sigma}_{\mathbf{X}}\right|}{|\mathbf{D}|} .
$$

\section{Remark:}

1) $\mathbf{D}\left(\boldsymbol{\Pi}-\left(\mathbf{D}+\boldsymbol{\Sigma}_{\mathbf{N}}\right)^{-1}\right) \mathbf{D}$ is positive semidefinite if and only if $\boldsymbol{\Pi}-\left(\mathbf{D}+\boldsymbol{\Sigma}_{\mathbf{N}}\right)^{-1}$ is positive semidefinite. Moreover, if $\boldsymbol{\Pi}-\left(\mathbf{D}+\boldsymbol{\Sigma}_{\mathbf{N}}\right)^{-1}$ is positive semidefinite, then $\boldsymbol{\Pi}$ is positive definite.

2) It follows by the Woodbury matrix identity that

$$
\left(\mathbf{D}^{-1}+\boldsymbol{\Sigma}_{\mathbf{N}}^{-1}\right)^{-1}=\mathbf{D}-\mathbf{D}\left(\mathbf{D}+\boldsymbol{\Sigma}_{\mathbf{N}}\right)^{-1} \mathbf{D} .
$$

Therefore, the diagonal entries of $\mathbf{D}\left(\boldsymbol{\Pi}-\left(\mathbf{D}+\boldsymbol{\Sigma}_{\mathbf{N}}\right)^{-1}\right) \mathbf{D}$ are the same as those of $\left(\mathbf{D}^{-1}+\boldsymbol{\Sigma}_{\mathbf{N}}^{-1}\right)^{-1}$ if and only if the diagonal entries of DID are the same as those of $\mathbf{D}$, i.e.,

$$
\sum_{j=1}^{\ell} d_{i, j}^{2} \pi_{j}=d_{i, i}, \quad i=1,2, \ldots, \ell .
$$

Since $d_{i, i}=d_{i}$ for all $i$ if $\mathbf{D} \in \mathbb{D}\left(\mathbf{d}, \boldsymbol{\Sigma}_{\mathbf{X}}\right)$, we can write (13) compactly as

$$
(\mathbf{D} \odot \mathbf{D}) \boldsymbol{\pi}=\mathbf{d}
$$

where $\odot$ is the Hadamard product and $\boldsymbol{\pi}=\left(\pi_{1}, \pi_{2}, \ldots, \pi_{\ell}\right)^{T}$. In view of the fact that the Hadamard product of two positive definite matrices is also positive definite, we have

$$
\boldsymbol{\pi}=(\mathbf{D} \odot \mathbf{D})^{-1} \mathbf{d} .
$$

Note that $\boldsymbol{\Pi}$ is uniquely determined by $\mathbf{D}$ and does not depend on $\boldsymbol{\Sigma}_{\mathbf{N}}$. However, $\boldsymbol{\Pi}-\left(\mathbf{D}+\boldsymbol{\Sigma}_{\mathbf{N}}\right)^{-1}$ is not guaranteed to be positive semidefinite. Therefore, if possible, we should choose $\boldsymbol{\Sigma}_{\mathbf{N}} \in \mathbb{N}\left(\boldsymbol{\Sigma}_{\mathbf{X}}\right)$ such that $\boldsymbol{\Pi}-\left(\mathbf{D}+\boldsymbol{\Sigma}_{\mathbf{N}}\right)^{-1}$ is positive semidefinite.

Proof: See Appendix C.

The following result shows that in the high-resolution regime, the desired $\Pi$ always exists.

Theorem 5: There exists an $\epsilon>0$ such that if $\|\mathbf{d}\| \leq \epsilon$, then

$$
R^{\star}(\mathbf{d})=\frac{1}{2} \log \frac{\left|\mathbf{\Sigma}_{\mathbf{X}}\right|}{|\mathbf{D}|}
$$

for $\mathbf{D} \in \mathbb{D}\left(\mathbf{d}, \boldsymbol{\Sigma}_{\mathbf{X}}\right)$.

Remark: It was shown in [25] that the Berger-Tung upper bound is asymptotically tight in the high-resolution regime for general sources. Our result indicates that if the source is Gaussian with a positive definite covariance matrix, then the 
Berger-Tung upper bound is tight when the distortion constraint is set below a certain threshold.

Proof: See Appendix D.

Now we shall impose some constraints so that $\left(\mathbf{P}_{2}\right)$ and $\left(\mathbf{P}_{2}^{\prime}\right)$ can be solved explicitly.

Lemma 5: If there exist $\mathbf{D} \in \mathbb{D}\left(\mathbf{d}, \boldsymbol{\Sigma}_{\mathbf{X}}\right)$ and $\boldsymbol{\Sigma}_{\mathbf{N}} \in \mathbb{N}\left(\boldsymbol{\Sigma}_{\mathbf{X}}\right)$ satisfying the following properties:

1) $\mathbf{D}+\boldsymbol{\Sigma}_{\mathbf{N}}$ is a diagonal matrix,

2) there exists a positive semidefinite diagonal matrix $\Delta$ such that the diagonal entries of $\mathbf{D} \Delta \mathbf{D}$ are the same as those of $\left(\mathbf{D}^{-1}+\boldsymbol{\Sigma}_{\mathbf{N}}^{-1}\right)^{-1}$,

then we have

$$
R^{\star}(\mathbf{d})=\underline{R}^{\star}\left(\mathbf{d}, \boldsymbol{\Sigma}_{\mathbf{N}}\right)=\bar{R}^{\star}\left(\mathbf{d}, \boldsymbol{\Sigma}_{\mathbf{N}}\right)=\frac{1}{2} \log \frac{\left|\boldsymbol{\Sigma}_{\mathbf{X}}\right|}{|\mathbf{D}|} .
$$

Proof: If $\mathbf{D}+\boldsymbol{\Sigma}_{\mathbf{N}}$ is a diagonal matrix, then the existence of a diagonal matrix $\boldsymbol{\Pi}$ such that $\mathbf{D}\left(\boldsymbol{\Pi}-\left(\mathbf{D}+\boldsymbol{\Sigma}_{\mathbf{N}}\right)^{-1}\right) \mathbf{D}$ is a positive semidefinite matrix with the diagonal entries the same as those of $\left(\mathbf{D}^{-1}+\boldsymbol{\Sigma}_{\mathbf{N}}^{-1}\right)^{-1}$ is equivalent to the existence of a positive semidefinite diagonal matrix $\Delta$ such that the diagonal entries of $\mathbf{D} \boldsymbol{\Delta} \mathbf{D}$ are the same as those of $\left(\mathbf{D}^{-1}+\boldsymbol{\Sigma}_{\mathbf{N}}^{-1}\right)^{-1}$. The proof is complete by invoking Lemma 4 .

The following result was first derived in [22] by coupling the Gaussian two-terminal source coding problem to a Gaussian CEO problem. Here we give an alternative proof using Lemma 5.

Theorem 6: For $\boldsymbol{\Sigma}_{\mathbf{X}}$ of the form

$$
\boldsymbol{\Sigma}_{\mathbf{X}}=\left(\begin{array}{ll}
1 & \rho \\
\rho & 1
\end{array}\right)
$$

with $\rho \in(0,1)$, we have

$$
R^{\star}(\mathbf{d})=\frac{1}{2} \log \left(\frac{\left(1-\rho^{2}\right)}{2 d_{1} d_{2}}\left(1+\sqrt{1+\frac{4 \rho^{2} d_{1} d_{2}}{\left(1-\rho^{2}\right)^{2}}}\right)\right)
$$

if $\max \left(d_{1}, d_{2}\right) \leq \min \left(1, \rho^{2} \min \left(d_{1}, d_{2}\right)+1-\rho^{2}\right)$.

Remark: It is easy to show that $R^{\star}(\mathbf{d})=$ $-\frac{1}{2} \log \left(\min \left(d_{1}, d_{2}\right)\right)$ if the condition $\max \left(d_{1}, d_{2}\right) \leq$ $\min \left(1, \rho^{2} \min \left(d_{1}, d_{2}\right)+1-\rho^{2}\right)$ is not satisfied.

Proof: It can be shown that $\mathbb{D}\left(\mathbf{d}, \boldsymbol{\Sigma}_{\mathbf{X}}\right)$ is nonempty if $\max \left(d_{1}, d_{2}\right) \leq \min \left(1, \rho^{2} \min \left(d_{1}, d_{2}\right)+1-\rho^{2}\right)$; specifically, we have

$$
\mathbf{D}=\left(\begin{array}{cc}
d_{1} & \theta \sqrt{d_{1} d_{2}} \\
\theta \sqrt{d_{1} d_{2}} & d_{2}
\end{array}\right) \in \mathbb{D}\left(\mathbf{d}, \boldsymbol{\Sigma}_{\mathbf{X}}\right)
$$

where

$$
\theta=\frac{\sqrt{\left(1-\rho^{2}\right)^{2}+4 \rho^{2} d_{1} d_{2}}-\left(1-\rho^{2}\right)}{2 \rho \sqrt{d_{1} d_{2}}} .
$$

Note that $\mathbf{D} \in \mathbb{D}\left(\mathbf{d}, \boldsymbol{\Sigma}_{\mathbf{X}}\right)$ implies that $\mathbf{D}^{-1}-\boldsymbol{\Sigma}_{\mathbf{X}}^{-1}$ is a positive semidefinite diagonal matrix.
Now we choose

$$
\boldsymbol{\Sigma}_{\mathbf{N}}=\left(\begin{array}{cc}
d_{1} & -\theta \sqrt{d_{1} d_{2}} \\
-\theta \sqrt{d_{1} d_{2}} & d_{2}
\end{array}\right)
$$

It can be verified that

$$
\begin{aligned}
\mathbf{D}+\boldsymbol{\Sigma}_{\mathbf{N}} & =\left(\begin{array}{cc}
2 d_{1} & 0 \\
0 & 2 d_{2}
\end{array}\right) \\
\mathbf{D}^{-1}+\boldsymbol{\Sigma}_{\mathbf{N}}^{-1} & =\left(\begin{array}{cc}
\frac{2}{\left(1-\theta^{2}\right) d_{1}} & 0 \\
0 & \frac{2}{\left(1-\theta^{2}\right) d_{2}}
\end{array}\right) .
\end{aligned}
$$

Since both $\boldsymbol{\Sigma}_{\mathbf{X}}^{-1}-\mathbf{D}^{-1}$ and $\mathbf{D}^{-1}+\boldsymbol{\Sigma}_{\mathbf{N}}^{-1}$ are diagonal matrices, it follows that $\boldsymbol{\Sigma}_{\mathbf{N}} \in \mathbb{N}\left(\boldsymbol{\Sigma}_{\mathbf{X}}\right)$.

Let $\Delta$ be a diagonal matrix such that the diagonal entries of $\mathbf{D} \Delta \mathbf{D}$ are the same as those of $\left(\mathbf{D}^{-1}+\boldsymbol{\Sigma}_{\mathbf{N}}^{-1}\right)^{-1}$. We must have

$$
\boldsymbol{\Delta}=\left(\begin{array}{cc}
\frac{1-\theta^{2}}{2\left(1+\theta^{2}\right) d_{1}} & 0 \\
0 & \frac{1-\theta^{2}}{2\left(1+\theta^{2}\right) d_{2}}
\end{array}\right) \text {. }
$$

It is easy to see that $\Delta$ is positive semidefinite. The proof is complete by invoking Lemma 5 .

Note that $\mathbf{D}^{-1}+\boldsymbol{\Sigma}_{\mathbf{N}}^{-1}$ is a diagonal matrix if $\mathbf{D} \in \mathbb{D}\left(\mathbf{d}, \boldsymbol{\Sigma}_{\mathbf{X}}\right)$ and $\boldsymbol{\Sigma}_{\mathbf{N}} \in \mathbb{N}\left(\boldsymbol{\Sigma}_{\mathbf{X}}\right)$. Moreover, Lemma 5 requires that $\mathbf{D}+$ $\boldsymbol{\Sigma}_{\mathbf{N}}$ is also a diagonal matrix. Although this is possible in the two-terminal case as shown in the proof of Theorem 6, the existence of such kind of matrices is not obvious when $\ell>2$. The following result shows that this kind of matrices do exist; however, they must satisfy certain structural constraints.

Lemma 6: For a positive definite matrix $\mathbf{D}$ that cannot be converted to a block diagonal matrix (with more than one block) via permutation, ${ }^{1}$ if there exists a positive definite matrix $\boldsymbol{\Sigma}_{\mathbf{N}}$ such that both $\mathbf{D}+\boldsymbol{\Sigma}_{\mathbf{N}}$ and $\mathbf{D}^{-1}+\boldsymbol{\Sigma}_{\mathbf{N}}^{-1}$ are diagonal matrices, then $\mathbf{D}$ can be written as

$$
\mathbf{D}=\mathbf{A}^{\frac{1}{2}} \mathbf{U} \operatorname{diag}\left(r:(1+\lambda)^{-1},\left(1+\lambda^{-1}\right)^{-1}\right) \mathbf{U}^{T} \mathbf{A}^{\frac{1}{2}}
$$

for some positive definite diagonal matrix $\mathbf{A}$, unitary matrix $\mathbf{U}$, positive number $\lambda$, and integer $r \in[0, \ell]$. Here we use $\operatorname{diag}(r$ : $a, b)$ to denote a diagonal matrix such that the first $r$ diagonal entries are equal to $a$ and the rest of diagonal entries are equal to $b$.

Conversely, if $\mathbf{D}$ can be written in the form of (14), then there exists a positive definite matrix $\boldsymbol{\Sigma}_{\mathbf{N}}$ such that both $\mathbf{D}+\boldsymbol{\Sigma}_{\mathbf{N}}$ and $\mathbf{D}^{-1}+\boldsymbol{\Sigma}_{\mathbf{N}}^{-1}$ are diagonal matrices.

Proof: See Appendix E.

Theorem 7: If there exists $\mathbf{D} \in \mathbb{D}\left(\mathbf{d}, \boldsymbol{\Sigma}_{\mathbf{X}}\right)$ satisfying the following properties:

1) $\mathbf{D}$ can be written as

$$
\mathbf{D}=\mathbf{A}^{\frac{1}{2}} \mathbf{U} \operatorname{diag}\left(r:(1+\lambda)^{-1},\left(1+\lambda^{-1}\right)^{-1}\right) \mathbf{U}^{T} \mathbf{A}^{\frac{1}{2}}
$$

${ }^{1}$ That is to say, there does not exist a permutation matrix $\mathbf{P}$ such that $\mathbf{P} \boldsymbol{\Sigma}_{\mathbf{X}} \mathbf{P}^{T}$ is a block diagonal matrix. Note that if $\mathbf{D} \in \mathbb{D}\left(\boldsymbol{\Sigma}_{\mathbf{X}}\right)$ and $\mathbf{D}$ can be converted to a block diagonal matrix via permutation, then $\boldsymbol{\Sigma}_{\mathbf{X}}$ itself can also be converted to a block diagonal matrix via permutation. This implies that the source contains several groups of independent components, which can be treated separately. 
for some positive definite diagonal matrix $\mathbf{A}$, unitary matrix $\mathbf{U}$, positive number $\lambda$, and integer $r \in[0, \ell]$,

2) there exists a positive semidefinite diagonal matrix $\Delta$ such that the diagonal entries of

$\mathbf{U d i a g}\left(r:(1+\lambda)^{-1},\left(1+\lambda^{-1}\right)^{-1}\right) \mathbf{U}^{T}$

$$
\boldsymbol{\Delta} \mathbf{U d i a g}\left(r:(1+\lambda)^{-1},\left(1+\lambda^{-1}\right)^{-1}\right) \mathbf{U}^{T}
$$

are all equal to one,

then we have

$$
R^{\star}(\mathbf{d})=\frac{1}{2} \log \frac{\left|\boldsymbol{\Sigma}_{\mathbf{X}}\right|}{|\mathbf{D}|}
$$

Proof: See Appendix F.

Now we proceed to characterize $R^{\star}(\mathbf{d})$ for $\boldsymbol{\Sigma}_{\mathbf{X}}$ with certain special structures. First we need the following result, which is known as the Sherman-Morrison formula.

Lemma 7: Let $\boldsymbol{\Sigma}=\boldsymbol{\Delta}+a \boldsymbol{\mu} \boldsymbol{\mu}^{T}$, where $a \in \mathbb{R}$, $\boldsymbol{\mu}=\left(\mu_{1}, \mu_{2}, \ldots, \mu_{\ell}\right)^{T}$, and $\boldsymbol{\Delta}=\operatorname{diag}\left(\delta_{1}, \delta_{2}, \ldots, \delta_{\ell}\right)$ with $\delta_{i} \neq 0, i=1,2, \ldots, \ell$. We have

$$
\boldsymbol{\Sigma}^{-1}=\boldsymbol{\Delta}^{-1}+b \boldsymbol{\nu} \boldsymbol{\nu}^{T}
$$

if $1+a \sum_{i=1}^{\ell} \mu_{i}^{2} \delta_{i}^{-1} \neq 0$, where

$$
b=-\frac{a}{1+a \sum_{i=1}^{\ell} \mu_{i}^{2} \delta_{i}^{-1}}
$$

and $\boldsymbol{\nu}=\left(\nu_{1}, \nu_{2}, \ldots, \nu_{\ell}\right)^{T}$ with $\nu_{i}=\mu_{i} \delta_{i}^{-1}, i=1,2, \ldots, \ell$. Furthermore, the determinant of $\boldsymbol{\Sigma}$ is given by

$$
|\boldsymbol{\Sigma}|=\left(1+a \sum_{i=1}^{\ell} \mu_{i}^{2} \delta_{i}^{-1}\right) \prod_{i=1}^{\ell} \delta_{i}
$$

Consider $\boldsymbol{\Sigma}_{\mathbf{X}}$ of the form

$$
\boldsymbol{\Sigma}_{\mathbf{X}}=\boldsymbol{\Delta}_{\mathbf{X}}+\boldsymbol{\alpha} \boldsymbol{\alpha}^{T}
$$

where $\boldsymbol{\Delta}_{\mathbf{X}}=\operatorname{diag}\left(\delta_{\mathbf{X}, 1}, \delta_{\mathbf{X}, 2}, \ldots, \delta_{\mathbf{X}, \ell}\right)$ is a positive definite diagonal matrix and $\boldsymbol{\alpha}=\left(\alpha_{1}, \alpha_{2}, \ldots, \alpha_{\ell}\right)^{T}$ is a column vector. It follows by (15) in Lemma 7 that

$$
\boldsymbol{\Sigma}_{\mathbf{X}}^{-1}=\Delta_{\mathbf{X}}^{-1}-\beta \beta^{T}
$$

where $\boldsymbol{\beta}=\left(\beta_{1}, \beta_{2}, \ldots, \beta_{\ell}\right)^{T}$ with

$$
\beta_{i}=\frac{\alpha_{i}}{\delta_{\mathbf{X}, i} \sqrt{1+\sum_{j=1}^{\ell} \alpha_{j}^{2} \delta_{\mathbf{X}, j}^{-1}}}, \quad i=1,2, \ldots, \ell
$$

For $\mathbf{D} \in \mathbb{D}\left(\mathbf{d}, \boldsymbol{\Sigma}_{\mathbf{X}}\right)$, since $\mathbf{D}^{-1}-\boldsymbol{\Sigma}_{\mathbf{X}}^{-1}$ is a positive semidefinite diagonal matrix, it follows that we can write $\mathbf{D}^{-1}$ in the form of

$$
\mathrm{D}^{-1}=\boldsymbol{\Delta}_{\mathbf{D}}^{-1}-\boldsymbol{\beta} \boldsymbol{\beta}^{T}
$$

where $\boldsymbol{\Delta}_{\mathbf{D}}=\operatorname{diag}\left(\delta_{\mathbf{D}, 1}, \delta_{\mathbf{D}, 2}, \ldots, \delta_{\mathbf{D}, \ell}\right)$ is a positive definite diagonal matrix.

Similarly, for $\boldsymbol{\Sigma}_{\mathbf{X}}$ of the form

$$
\boldsymbol{\Sigma}_{\mathbf{X}}=\Delta_{\mathbf{X}}-\boldsymbol{\alpha} \boldsymbol{\alpha}^{T}
$$

we have

$$
\boldsymbol{\Sigma}_{\mathbf{X}}^{-1}=\boldsymbol{\Delta}_{\mathbf{X}}^{-1}+\boldsymbol{\beta}^{\prime}\left(\boldsymbol{\beta}^{\prime}\right)^{T}
$$

where $\beta^{\prime}=\left(\beta_{1}^{\prime}, \beta_{2}^{\prime}, \ldots, \beta_{\ell}^{\prime}\right)^{T}$ with

$$
\beta_{i}^{\prime}=\frac{\alpha_{i}}{\delta_{\mathbf{X}, i} \sqrt{1-\sum_{j=1}^{\ell} \alpha_{j}^{2} \delta_{\mathbf{X}, j}^{-1}}}, \quad i=1,2, \ldots, \ell .
$$

Note that $\boldsymbol{\beta}^{\prime}$ is well defined since by (16) and (19), we must have $1-\sum_{j=1}^{\ell} \alpha_{j}^{2} \delta_{\mathbf{X}, j}^{-1} \in(0,1]$. For $\mathbf{D} \in \mathbb{D}\left(\mathbf{d}, \boldsymbol{\Sigma}_{\mathbf{X}}\right)$, we can write $\mathrm{D}^{-1}$ in the form of

$$
\mathbf{D}^{-1}=\boldsymbol{\Delta}_{\mathbf{D}}^{-1}+\boldsymbol{\beta}^{\prime}\left(\boldsymbol{\beta}^{\prime}\right)^{T}
$$

where $\boldsymbol{\Delta}_{\mathbf{D}}=\operatorname{diag}\left(\delta_{\mathbf{D}, 1}, \delta_{\mathbf{D}, 2}, \ldots, \delta_{\mathbf{D}, \ell}\right)$ is a positive definite diagonal matrix.

Note that for $\left(X_{1}, X_{2}, \ldots, X_{\ell}\right)^{T}$ with covariance matrix $\boldsymbol{\Sigma}_{\mathbf{X}}$ of the form (17), one can write $X_{i}=\alpha_{i} X_{0}+\sqrt{\delta_{\mathbf{X}, i}} \tilde{X}_{i}, i=$ $1,2, \ldots, \ell$, where $X_{0}, \tilde{X}_{1}, \ldots, \tilde{X}_{\ell}$ are independent zero-mean unit-variance Gaussian random variables. However, this interpretation does not apply to $\boldsymbol{\Sigma}_{\mathbf{X}}$ of the form (19) in general.

Now we are ready to state the following result.

\section{Theorem 8:}

1) For $\boldsymbol{\Sigma}_{\mathbf{X}}$ of the form of (17), we have

$$
R^{\star}(\mathbf{d})=\frac{1}{2} \log \frac{\left|\boldsymbol{\Sigma}_{\mathbf{X}}\right|}{|\mathbf{D}|}
$$

if there exists $\mathbf{D} \in \mathbb{D}\left(\mathbf{d}, \boldsymbol{\Sigma}_{\mathbf{X}}\right)$ such that

$$
1+\sum_{j=1}^{\ell} \frac{v_{j}^{2}\left(v_{j}^{2}-v_{i}^{2}\right)}{1+2 v_{j}^{2}} \geq 0, \quad i=1,2, \ldots, \ell
$$

where

$$
v_{i}=\frac{\beta_{i} \delta_{\mathbf{D}, i}^{\frac{1}{2}}}{\sqrt{1-\sum_{j=1}^{\ell} \beta_{j}^{2} \delta_{\mathbf{D}, j}}}, \quad i=1,2, \ldots, \ell .
$$

2) For $\boldsymbol{\Sigma}_{\mathbf{X}}$ of the form of (19), we have

$$
R^{\star}(\mathbf{d})=\frac{1}{2} \log \frac{\left|\boldsymbol{\Sigma}_{\mathbf{X}}\right|}{|\mathbf{D}|}
$$

if there exists $\mathbf{D} \in \mathbb{D}\left(\mathbf{d}, \boldsymbol{\Sigma}_{\mathbf{X}}\right)$ such that

$$
\begin{aligned}
& 1+\sum_{j=1}^{\ell} \frac{\left(v_{j}^{\prime}\right)^{2}\left(\left(v_{j}^{\prime}\right)^{2}-\left(v_{i}^{\prime}\right)^{2}\right)}{1-2\left(v_{j}\right)^{2}} \geq 0, \quad i=1,2, \ldots, \ell \\
& 1-2\left(v_{i}^{\prime}\right)^{2}>0, \quad i=1,2, \ldots, \ell
\end{aligned}
$$


where

$$
v_{i}^{\prime}=\frac{\beta_{i}^{\prime} \delta_{\mathbf{D}, i}^{\frac{1}{2}}}{\sqrt{1+\sum_{j=1}^{\ell}\left(\beta_{j}^{\prime}\right)^{2} \delta_{\mathbf{D}, j}}}, \quad i=1,2, \ldots, \ell
$$

Remark: The conditions (20), (21), and (22) in Theorem 8 are not always satisfied. Consider the following example. For $\boldsymbol{\Sigma}_{\mathbf{X}}$ of the form (17), set $\boldsymbol{\Delta}_{\mathbf{X}}=\mathbf{I}$ and $\boldsymbol{\alpha}=\left(\alpha_{1}, \alpha_{2}, \ldots, \alpha_{\ell}\right)$ with $\alpha_{1}=$ $\cdots=\alpha_{\ell-1}=1$ and $\alpha_{\ell}=\sqrt{2}$. We have $\boldsymbol{\beta}=\left(\beta_{1}, \beta_{2}, \ldots, \beta_{\ell}\right)$, where $\beta_{1}=\cdots=\beta_{\ell-1}=\sqrt{\frac{1}{\ell+2}}$ and $\beta_{\ell}=\sqrt{\frac{2}{\ell+2}}$. Now let $\mathbf{D}=\left(\boldsymbol{\Delta}_{\mathbf{D}}^{-1}-\boldsymbol{\beta} \boldsymbol{\beta}^{T}\right)^{-1}$, where $\boldsymbol{\Delta}_{\mathbf{D}}=\operatorname{diag}\left(\delta_{\mathbf{D}}, \delta_{\mathbf{D}}, \ldots, \delta_{\mathbf{D}}\right)$ with $\delta_{\mathbf{D}}=\frac{\ell}{\ell+1}$. Furthermore, let $\mathbf{d}=\left(d_{1,1}, d_{2,2}, \ldots, d_{\ell, \ell}\right)^{T}$. It is clear that $\mathbf{D} \in \mathbb{D}\left(\mathbf{d}, \boldsymbol{\Sigma}_{\mathbf{X}}\right)$. Note that $v_{i}=\sqrt{\frac{\ell}{2(\ell+1)}}, i=$ $1, \ldots, \ell-1$, and $v_{\ell}=\sqrt{\frac{\ell}{\ell+1}}$. Therefore, we have

$$
1+\sum_{j=1}^{\ell} \frac{v_{j}^{2}\left(v_{j}^{2}-v_{l}^{2}\right)}{1+2 v_{j}^{2}}=1-\frac{\ell^{2}(\ell-1)}{4(\ell+1)(2 \ell+1)}
$$

which converges to $-\infty$ as $\ell \rightarrow \infty$.

Proof: See Appendix G.

Corollary 2: For $\boldsymbol{\Sigma}_{\mathbf{X}}$ of the form

$$
\boldsymbol{\Sigma}_{\mathbf{X}}=\left(\begin{array}{cccc}
1 & \rho & \cdots & \rho \\
\rho & 1 & \cdots & \rho \\
\vdots & \vdots & \ddots & \vdots \\
\rho & \rho & \cdots & 1
\end{array}\right)
$$

with $\rho \in\left(-\frac{1}{\ell-1}, 1\right)$ and $\mathbf{d}=(d, d, \ldots, d)^{T}$ with $d \in(0,1]$, we have

$$
R^{\star}(\mathbf{d})=\frac{1}{2} \log \frac{(1-\rho)^{\ell-1}(1-\rho+\ell \rho)}{d^{\ell}(1-\theta)^{\ell-1}(1-\theta+\ell \theta)}
$$

where

$$
\theta=\frac{\rho}{1+\kappa(1-\rho)(1-\rho+\ell \rho)}
$$

with $\kappa$ being the unique nonnegative solution to

$$
\frac{1-\rho}{1+\kappa(1-\rho)}\left(1+\frac{\rho}{1-\rho+\kappa(1-\rho)(1-\rho+\ell \rho)}\right)=d
$$

Remark: The case $\rho \in(0,1)$ was first solved in [22].

Proof: Consider D of the form

$$
\mathbf{D}^{-1}=\boldsymbol{\Sigma}_{\mathbf{X}}^{-1}+\kappa \mathbf{I}
$$

where $\kappa \geq 0$. It is clear that $\mathbf{D} \in \mathbb{D}\left(\boldsymbol{\Sigma}_{\mathbf{X}}\right)$. It can be verified that

$$
\begin{aligned}
\mathbf{D}=\frac{1-\rho}{1+\kappa(1-\rho)} & \\
& \times\left(\mathbf{I}+\frac{\rho}{1-\rho+\kappa(1-\rho)(1-\rho+\ell \rho)} \mathbf{1 1}^{T}\right) .
\end{aligned}
$$

Note that for each $d \in(0,1]$, there exists a unique $\kappa \geq 0$ such that $d_{i, i}=d$ for all $i$. Moreover, we have

$$
\frac{1}{2} \log \frac{\left|\boldsymbol{\Sigma}_{\mathbf{X}}\right|}{|\mathbf{D}|}=\frac{1}{2} \log \frac{(1-\rho)^{\ell-1}(1-\rho+\ell \rho)}{d^{\ell}(1-\theta)^{\ell-1}(1-\theta+\ell \theta)}
$$

where

$$
\theta=\frac{\rho}{1+\kappa(1-\rho)(1-\rho+\ell \rho)}
$$

Since $\boldsymbol{\Sigma}_{\mathbf{X}}$ can be written in the form of (17) when $\rho \in[0,1)$ and in the form of (19) when $\rho \in\left(-\frac{1}{\ell-1}, 0\right)$, the proof can be readily completed by verifying that the conditions in Theorem 8 are satisfied.

\section{CONCLUSION}

A unified method for deriving the lower bound on the sum rate of the direct and indirect Gaussian multiterminal source coding problems is presented. In contrast with the existing proofs, the estimation-theoretic nature of this new method allows us to circumvent Shannon's entropy power inequality. Note that the application of this method is not restricted to scalar sources studied in this work. Indeed, it is not hard to see that the new method is equally suitable for vector Gaussian sources. In fact, the advantage of the new method becomes more evident in the vector case since in that setting Shannon's entropy power inequality either yields suboptimal bounds or requires the enhancement technique introduced in [23].

\section{APPENDIX A}

\section{ProOF OF THEOREM 3}

We need the following result from [14], [15], which is a generalization of the well-known intermediate value theorem.

Lemma 8: If $\phi_{1}, \phi_{2}, \ldots, \phi_{\ell}$ are continuous in an $\ell$-dimensional rectangle $\left\{\mathbf{c}=\left(c_{1}, c_{2}, \ldots, c_{\ell}\right)^{T}: a_{i} \leq c_{i} \leq b_{i}, i=\right.$

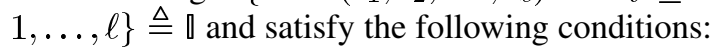

1) $\phi_{i}(\mathbf{c}) \leq 0$ for each $\mathbf{c} \in \mathbb{\|}$ with $c_{i}=a_{i}, i=1,2, \ldots, \ell$,

2) $\phi_{i}(\mathbf{c}) \geq 0$ for each $\mathbf{c} \in \mathbb{\text { with }} c_{i}=b_{i}, i=1,2, \ldots, \ell$, then there exists at least one point $\mathbf{c}^{*} \in \llbracket$ such that $\phi_{i}\left(\mathbf{c}^{*}\right)=0$ for all $i$.

Now we proceed to prove Theorem 3 . Let $U_{\mathcal{L}}$ be generated by $X_{\mathcal{L}}$ through parallelled Gaussian test channels, i.e., $U_{i}=$ $c_{i} X_{i}+Z_{i}, i=1,2, \ldots, \ell$, where $c_{i} \in[0, \infty)$ and $Z_{i}$ is a zero-mean unit-variance Gaussian random variable independent of everything else. Define

$$
\phi_{i}(\mathbf{c})=d_{i}-\mathbf{D}_{X_{i} \mid\left(U_{j}\right)_{j \neq i}}, \quad i=1,2, \ldots, \ell .
$$

It is clear that $\phi_{1}, \phi_{2}, \ldots, \phi_{\ell}$ are continuous functions of $\mathbf{c}$. We set

$$
\begin{aligned}
a_{i} & =0, \quad i=1,2, \ldots, \ell \\
b_{i} & =\sqrt{\frac{\sigma_{X_{i}}^{2}-d_{i}}{\sigma_{X_{i}}^{2} d_{i}}}, \quad i=1,2, \ldots, \ell .
\end{aligned}
$$


Note that

$$
\phi_{i}(\mathbf{c}) \leq d_{i}-\mathbf{D}_{X_{i} \mid\left(X_{j}\right)_{j \neq i}} \leq 0
$$

if $c_{i}=a_{i}$ and $d_{i} \leq \mathbf{D}_{X_{i} \mid\left(X_{j}\right)_{j \neq i}}, i=1,2, \ldots, \ell$. Moreover, we have

$$
\phi_{i}(\mathbf{c}) \geq d_{i}-\mathbf{D}_{X_{i} \mid U_{i}}=0
$$

if $c_{i}=b_{i}, i=1,2, \ldots, \ell$. The proof is complete by invoking Lemma 8 .

\section{APPENDIX B \\ PROOF OF THEOREM 4}

The following lemma is easy to verify.

Lemma 9: Let $\mathbf{S}=\operatorname{diag}\left(s_{1}, s_{2}, \ldots, s_{\ell}\right)$ and $\mathbf{s}=$ $\left(s_{1}, s_{2}, \ldots, s_{\ell}\right)^{T}$. For any $\ell \times \ell$ matrices $\mathbf{A}$ and $\mathbf{B}$, we have

$$
(\mathbf{A S B})_{i, i}=\left(\left(\mathbf{A} \odot \mathbf{B}^{T}\right) \mathbf{s}\right)_{i}, \quad i=1,2, \ldots, \ell
$$

where $(\mathbf{A S B})_{i, i}$ is the $i$ th diagonal entry of ASB, $\left(\left(\mathbf{A} \odot \mathbf{B}^{T}\right) \mathbf{s}\right)_{i}$ is the $i$ th entry of $\left(\mathbf{A} \odot \mathbf{B}^{T}\right) \mathbf{s}$, and $\odot$ is the Hadamard product.

Theorem 4 is a simple consequence of the following result.

Lemma 10: Let $\mathbf{A}$ be an $\ell \times \ell$ positive definite matrix. For any $\ell \times \ell$ diagonal matrix $\mathbf{S}$, if $\mathbf{A}+\mathbf{S}$ is positive definite and $\left((\mathbf{A}+\mathbf{S})^{-1}\right)_{i, i}=\left(\mathbf{A}^{-1}\right)_{i, i}$ for all $i$, then $\mathbf{S}=\mathbf{0}$.

Proof: Suppose there exists a nonzero diagonal matrix $\mathbf{S}$ such that $\mathbf{A}+\mathbf{S}$ is positive definite and $\left((\mathbf{A}+\mathbf{S})^{-1}\right)_{i, i}=$ $\left(\mathbf{A}^{-1}\right)_{i, i}$ for all $i$. First consider the case where $\mathbf{S}$ is nonsingular. Since

$$
(\mathbf{A}+\mathbf{S})^{-1}=\mathbf{A}^{-1}-\mathbf{A}^{-1}\left(\mathbf{A}^{-1}+\mathbf{S}^{-1}\right)^{-1} \mathbf{A}^{-1}
$$

it follows that

$$
\left(\mathbf{A}^{-1}\left(\mathbf{A}^{-1}+\mathbf{S}^{-1}\right)^{-1} \mathbf{A}^{-1}\right)_{i, i}=0, \quad i=1,2, \ldots, \ell .
$$

In view of the fact that

$$
\left(\mathbf{A}^{-1}+\mathbf{S}^{-1}\right)^{-1}=\mathbf{S}-\mathbf{S}(\mathbf{A}+\mathbf{S})^{-1} \mathbf{S}
$$

we have

$$
\begin{aligned}
& \mathbf{A}^{-1}\left(\mathbf{A}^{-1}+\mathbf{S}^{-1}\right)^{-1} \mathbf{A}^{-1} \\
&=\mathbf{A}^{-1} \mathbf{S} \mathbf{A}^{-1}-\mathbf{A}^{-1} \mathbf{S}(\mathbf{A}+\mathbf{S})^{-1} \mathbf{S} \mathbf{A}^{-1}
\end{aligned}
$$

Substituting (24) into (23) yields

$$
\begin{array}{r}
\left(\mathbf{A}^{-1} \mathbf{S} \mathbf{A}^{-1}\right)_{i, i}=\left(\mathbf{A}^{-1} \mathbf{S}(\mathbf{A}+\mathbf{S})^{-1} \mathbf{S A}^{-1}\right)_{i, i} \\
i=1,2, \ldots, \ell .
\end{array}
$$

It follows by Lemma 9 that

$$
\begin{aligned}
& \left(\mathbf{A}^{-1} \mathbf{S} \mathbf{A}^{-1}\right)_{i, i} \\
& \quad=\left(\left(\mathbf{A}^{-1} \odot \mathbf{A}^{-1}\right) \mathbf{s}\right)_{i} \\
& \left(\mathbf{A}^{-1} \mathbf{S}(\mathbf{A}+\mathbf{S})^{-1} \mathbf{S} \mathbf{A}^{-1}\right)_{i, i} \\
& \quad=\left(\left(\mathbf{A}^{-1} \odot\left(\mathbf{A}^{-1} \mathbf{S}(\mathbf{A}+\mathbf{S})^{-1}\right)\right) \mathbf{s}\right)_{i} .
\end{aligned}
$$

Therefore, we can write (25) alternatively as

$$
\left(\mathbf{A}^{-1} \odot\left(\mathbf{A}^{-1}-\mathbf{A}^{-1} \mathbf{S}(\mathbf{A}+\mathbf{S})^{-1}\right)\right) \mathbf{s}=\mathbf{0}
$$

which can be further written as

$$
\left(\mathbf{A}^{-1} \odot(\mathbf{A}+\mathbf{S})^{-1}\right) \mathbf{s}=\mathbf{0}
$$

due to the fact that $\mathbf{A}^{-1}-\mathbf{A}^{-1} \mathbf{S}(\mathbf{A}+\mathbf{S})^{-1}=(\mathbf{A}+\mathbf{S})^{-1}$. Since the Hadamard product of two positive definite matrices is also positive definite, we must have $\mathbf{s}=\mathbf{0}$, which results in a contradiction.

Now consider the case where $\mathbf{S}$ is singular. Without loss of generality, we shall write $\mathbf{S}=\operatorname{diag}\left(s_{1}, \ldots, s_{r}, 0, \ldots, 0\right)$, where $s_{i} \neq 0, i=1, \ldots, r$. Moreover, we write $\mathbf{A}$ in a partitioned form

$$
\mathbf{A}=\left(\begin{array}{ll}
\mathbf{A}_{1,1} & \mathbf{A}_{1,2} \\
\mathbf{A}_{1,2}^{T} & \mathbf{A}_{2,2}
\end{array}\right)
$$

where $\mathbf{A}_{1,1}$ is an $r \times r$ submatrix. Let $\tilde{\mathbf{S}}=\operatorname{diag}\left(s_{1}, \ldots, s_{r}\right)$. Note that

$$
\begin{aligned}
(\mathbf{A}+\mathbf{S})^{-1} & =\left(\begin{array}{cc}
\mathbf{A}_{1,1}+\tilde{\mathbf{S}} & \mathbf{A}_{1,2} \\
\mathbf{A}_{1,2}^{T} & \mathbf{A}_{2,2}
\end{array}\right)^{-1} \\
& =\left(\begin{array}{cc}
(\tilde{\mathbf{A}}+\tilde{\mathbf{S}})^{-1} & * \\
* & *
\end{array}\right) \\
\mathbf{A}^{-1} & =\left(\begin{array}{cc}
\tilde{\mathbf{A}}^{-1} & \# \\
\# & \#
\end{array}\right)
\end{aligned}
$$

where $\tilde{\mathbf{A}}=\mathbf{A}_{1,1}-\mathbf{A}_{1,2} \mathbf{A}_{2,2}^{-1} \mathbf{A}_{1,2}^{T}$. Therefore, we have $((\tilde{\mathbf{A}}+$ $\left.\tilde{\mathbf{S}})^{-1}\right)_{i, i}=\left(\tilde{\mathbf{A}}^{-1}\right)_{i, i}$ for all $i$. It can be readily shown that $\tilde{\mathbf{S}}=\mathbf{0}$ by invoking the previous argument.

Now we proceed to prove Theorem 4 . If there exist nonidentical $\mathbf{D}_{1}, \mathbf{D}_{2} \in \mathbb{D}\left(\mathbf{d}, \boldsymbol{\Sigma}_{\mathbf{X}}\right)$, then one can find a nonzero diagonal matrix $\mathbf{S}$ such that the diagonal entries of $\mathbf{D}_{1}=\left(\mathbf{D}_{2}^{-1}+\mathbf{S}\right)^{-1}$ are the same as those of $\mathbf{D}_{2}$, which is contradictory with Lemma 10. The proof is complete.

\section{APPENDIX C}

PROOF OF LEMMA 4

Consider the following convex semidefinite programming problem $^{2}$

$$
\begin{aligned}
\min _{\mathbf{D}, \gamma_{i}, i=1,2, \ldots, \ell} & -\log \left|\mathbf{D}+\mathbf{\Sigma}_{\mathbf{N}}\right|-\sum_{i=1}^{\ell} \log \gamma_{i} \quad\left(\mathbf{R P}_{2}\right) \\
\text { subject to } & \operatorname{diag}\left(\gamma_{1}, \gamma_{2}, \ldots, \gamma_{\ell}\right) \preceq\left(\mathbf{D}^{-1}+\mathbf{\Sigma}_{\mathbf{N}}^{-1}\right)^{-1} \\
& \mathbf{0} \mathbf{D} \\
& d_{i, i} \leq d_{i}, \quad i=1,2, \ldots, \ell \\
& 0<\gamma_{i}, \quad i=1,2, \ldots, \ell
\end{aligned}
$$

which is essentially a relaxed version of $\left(\mathbf{P}_{2}\right)$. Note that the constraint $d_{i, i} \leq d_{i}$ can be written as $\mathbf{E}_{i} \mathbf{D} \mathbf{E}_{i} \preceq d_{i} \mathbf{E}_{i}$, where $\mathbf{E}_{i}$

\footnotetext{
${ }^{2}$ To see the convexity of the function $\operatorname{diag}\left(\gamma_{1}, \gamma_{2}, \ldots, \gamma_{\ell}\right)-$ $\left(\mathbf{D}^{-1}+\Sigma_{\mathbf{N}}^{-1}\right)^{-1}$ in the first constraint, one can write $\left(\mathbf{D}^{-1}+\mathbf{\Sigma}_{\mathbf{N}}^{-1}\right)^{-1}=$ $\boldsymbol{\Sigma}_{\mathbf{N}}-\boldsymbol{\Sigma}_{\mathbf{N}}\left(\mathbf{D}+\boldsymbol{\Sigma}_{\mathbf{N}}\right)^{-1} \boldsymbol{\Sigma}_{\mathbf{N}}$ and invoke the fact that $\left(\mathbf{D}+\boldsymbol{\Sigma}_{\mathbf{N}}\right)^{-1}$ is matrix convex in $\mathbf{D}$ [3].
} 
is an all-zero matrix except that the $i$ th diagonal entry is equal to one. The Lagrangian of $\left(\mathbf{R P}_{2}\right)$ is given by

$$
\begin{aligned}
L\left(\mathbf{D}, \gamma_{1}, \gamma_{2}, \ldots, \gamma_{\ell}\right) \\
=-\log \left|\mathbf{D}+\boldsymbol{\Sigma}_{\mathbf{N}}\right|-\sum_{i=1}^{\ell} \log \gamma_{i} \\
\quad+\operatorname{tr}\left(\boldsymbol{\Lambda}\left(\operatorname{diag}\left(\gamma_{1}, \gamma_{2}, \ldots, \gamma_{\ell}\right)-\left(\mathbf{D}^{-1}+\boldsymbol{\Sigma}_{\mathbf{N}}^{-1}\right)^{-1}\right)\right) \\
\quad+\sum_{i=1}^{\ell} \operatorname{tr}\left(\mathbf{\Pi}_{i} \mathbf{E}_{i} \mathbf{D} \mathbf{E}_{i}\right)
\end{aligned}
$$

where $\Lambda, \Pi_{1}, \Pi_{2}, \ldots, \Pi_{\ell}$ are positive semidefinite matrices. Therefore, $\left(\mathbf{D}, \gamma_{1}, \gamma_{2}, \ldots, \gamma_{\ell}\right)$ is an optimal solution to $\left(\mathbf{R} \mathbf{P}_{2}\right)$ if it satisfies the following KKT conditions [24]:

$$
\begin{aligned}
& \nabla_{\mathbf{D}} L\left(\mathbf{D}, \gamma_{1}, \gamma_{2}, \ldots, \gamma_{\ell}\right)=\mathbf{0} \\
& \nabla_{\gamma_{i}} L\left(\mathbf{D}, \gamma_{1}, \gamma_{2}, \ldots, \gamma_{\ell}\right)=0, \quad i=1,2, \ldots, \ell \\
& \mathbf{0} \prec \mathbf{D} \\
& \mathbf{E}_{i} \mathbf{D} \mathbf{E}_{i} \preceq d_{i} \mathbf{E}_{i}, \quad i=1,2, \ldots, \ell \\
& 0<\gamma_{i}, \quad i=1,2, \ldots, \ell \\
& \boldsymbol{\Lambda}\left(\operatorname{diag}\left(\gamma_{1}, \gamma_{2}, \ldots, \gamma_{\ell}\right)-\left(\mathbf{D}^{-1}+\mathbf{\Sigma}_{\mathbf{N}}^{-1}\right)^{-1}\right)=\mathbf{0} \\
& \boldsymbol{\Pi}_{i}\left(\mathbf{E}_{i} \mathbf{D} \mathbf{E}_{i}-d_{i} \mathbf{E}_{i}\right)=\mathbf{0}, \quad i=1,2, \ldots, \ell .
\end{aligned}
$$

These KKT conditions can be rewritten as

$$
\begin{aligned}
& \mathbf{D}\left(\sum_{i=1}^{\ell} \mathbf{E}_{i} \boldsymbol{\Pi}_{i} \mathbf{E}_{i}-\left(\mathbf{D}+\mathbf{\Sigma}_{\mathbf{N}}\right)^{-1}\right) \mathbf{D} \\
& \quad=\left(\mathbf{D}^{-1}+\boldsymbol{\Sigma}_{\mathbf{N}}^{-1}\right)^{-1} \boldsymbol{\Lambda}\left(\mathbf{D}^{-1}+\boldsymbol{\Sigma}_{\mathbf{N}}^{-1}\right)^{-1} \\
& \gamma_{i}=\lambda_{i, i}^{-1}, \quad i=1,2, \ldots, \ell \\
& \mathbf{0} \prec \mathbf{D} \\
& \mathbf{E}_{i} \mathbf{D} \mathbf{E}_{i} \preceq d_{i} \mathbf{E}_{i}, \quad i=1,2, \ldots, \ell \\
& 0<\gamma_{i}, \quad i=1,2, \ldots, \ell \\
& \boldsymbol{\Lambda}\left(\operatorname{diag}\left(\gamma_{1}, \gamma_{2}, \ldots, \gamma_{\ell}\right)-\left(\mathbf{D}^{-1}+\mathbf{\Sigma}_{\mathbf{N}}^{-1}\right)^{-1}\right)=\mathbf{0} \\
& \boldsymbol{\Pi}_{i}\left(\mathbf{E}_{i} \mathbf{D} \mathbf{E}_{i}-d_{i} \mathbf{E}_{i}\right)=\mathbf{0}, \quad i=1,2, \ldots, \ell
\end{aligned}
$$

where $\lambda_{i, i}$ is the $i$ th diagonal entry of $\boldsymbol{\Lambda}$.

It is easy to see that $\mathbf{D}^{-1}+\boldsymbol{\Sigma}_{\mathbf{N}}^{-1}$ is a positive definite diagonal matrix if $\mathbf{D} \in \mathbb{D}\left(\mathbf{d}, \boldsymbol{\Sigma}_{\mathbf{X}}\right)$ and $\boldsymbol{\Sigma}_{\mathbf{N}} \in \mathbb{N}\left(\boldsymbol{\Sigma}_{\mathbf{X}}\right)$. Therefore, the conditions (28)-(32) are automatically satisfied if we choose $\mathbf{D}$ from $\mathbb{D}\left(\mathbf{d}, \boldsymbol{\Sigma}_{\mathbf{X}}\right)$ and set

$$
\operatorname{diag}\left(\gamma_{1}, \gamma_{2}, \ldots, \gamma_{\ell}\right)=\left(\mathbf{D}^{-1}+\mathbf{\Sigma}_{\mathbf{N}}^{-1}\right)^{-1}
$$

Furthermore, it is easy to see that $\sum_{i=1}^{\ell} \mathbf{E}_{i} \boldsymbol{\Pi}_{i} \mathbf{E}_{i}$ is a diagonal matrix. As a consequence, the existence of positive semidefinite matrices $\boldsymbol{\Lambda}, \boldsymbol{\Pi}_{1}, \boldsymbol{\Pi}_{2}, \ldots, \boldsymbol{\Pi}_{\ell}$ that satisfy (26) and (27) is equivalent to the existence of a diagonal matrix $\boldsymbol{\Pi}=\operatorname{diag}\left(\pi_{1}, \pi_{2}, \ldots, \pi_{\ell}\right)$ such that $\mathbf{D}\left(\boldsymbol{\Pi}-\left(\mathbf{D}+\boldsymbol{\Sigma}_{\mathbf{N}}\right)^{-1}\right) \mathbf{D}$ is a positive semidefinite matrix with the diagonal entries the same as those of $\left(\mathbf{D}^{-1}+\boldsymbol{\Sigma}_{\mathbf{N}}^{-1}\right)^{-1}$.
Note that given $\boldsymbol{\Sigma}_{\mathbf{N}} \in \mathbb{N}\left(\boldsymbol{\Sigma}_{\mathbf{X}}\right)$, the mapping $\operatorname{diag}\left(\gamma_{1}, \gamma_{2}, \ldots, \gamma_{\ell}\right)=\left(\mathbf{D}^{-1}+\boldsymbol{\Sigma}_{\mathbf{N}}^{-1}\right)^{-1}$ induces a one-to-one correspondence between $\left(\gamma_{1}, \gamma_{2}, \ldots, \gamma_{\ell}\right) \in\left(0, \sigma_{1}^{2}\right] \times\left(0, \sigma_{2}^{2}\right] \times \cdots\left(0, \sigma_{\ell}^{2}\right]$ and $\mathbf{D} \in \mathbb{D}\left(\boldsymbol{\Sigma}_{\mathbf{X}}\right)$. Therefore, our choice of $\left(\mathbf{D}, \gamma_{1}, \gamma_{2}, \ldots, \gamma_{\ell}\right)$ guarantees that the conditions $\mathbf{D} \preceq \boldsymbol{\Sigma}_{\mathbf{X}}$ and $\gamma_{i} \leq \sigma_{i}^{2}$, $i=1,2, \ldots, \ell$, are satisfied. As a consequence, if $\left(\mathbf{D}, \gamma_{1}, \gamma_{2}, \ldots, \gamma_{\ell}\right)$ is an optimal solution to $\left(\mathbf{R P}_{2}\right)$, then it is also an optimal solution to $\left(\mathbf{P}_{2}\right)$. The proof is complete by invoking Corollary 1.

\section{APPENDIX D \\ PROOF OF THEOREM 5}

First note that Theorem 3 implies that $\mathbb{D}\left(\mathbf{d}, \boldsymbol{\Sigma}_{\mathbf{X}}\right)$ is nonempty when $\|\mathbf{d}\|$ is sufficiently small. Now we choose $\mathbf{D} \in \mathbb{D}\left(\mathbf{d}, \boldsymbol{\Sigma}_{\mathbf{X}}\right)$, $\boldsymbol{\Sigma}_{\mathbf{N}} \in \mathbb{N}\left(\boldsymbol{\Sigma}_{\mathbf{X}}\right)$ and set

$$
\Delta=\left(\mathbf{D}^{-1}-\mathbf{\Sigma}_{\mathbf{X}}^{-1}\right)^{-1} .
$$

It is clear that $\Delta$ is a positive definite diagonal matrix and we have $\boldsymbol{\Delta} \rightarrow \mathbf{0}$ as $\|\mathbf{d}\| \rightarrow 0$. Using the Woodbury matrix identity, it can be readily verified that

$$
\begin{aligned}
\mathbf{D} & =\left(\boldsymbol{\Delta}^{-1}+\boldsymbol{\Sigma}_{\mathbf{X}}^{-1}\right)^{-1} \\
& =\boldsymbol{\Delta}^{\frac{1}{2}}\left(\mathbf{I}+\boldsymbol{\Delta}^{\frac{1}{2}} \boldsymbol{\Sigma}_{\mathbf{X}}^{-1} \boldsymbol{\Delta}^{\frac{1}{2}}\right)^{-1} \boldsymbol{\Delta}^{\frac{1}{2}} \\
& =\boldsymbol{\Delta}^{\frac{1}{2}}(\mathbf{I}-\mathbf{A}) \boldsymbol{\Delta}^{\frac{1}{2}}
\end{aligned}
$$

where

$$
\mathbf{A}=\left(a_{i, j}\right) \triangleq \boldsymbol{\Delta}^{\frac{1}{2}}\left(\boldsymbol{\Delta}+\boldsymbol{\Sigma}_{\mathbf{X}}\right)^{-1} \boldsymbol{\Delta}^{\frac{1}{2}} .
$$

Note that we have $\mathbf{A} \rightarrow \mathbf{0}$ as $\|\mathbf{d}\| \rightarrow 0$.

In view of Lemma 4 and its remark, we need to find a diagonal matrix $\boldsymbol{\Pi}$ such that $\boldsymbol{\Pi}-\left(\mathbf{D}+\boldsymbol{\Sigma}_{\mathbf{N}}\right)^{-1}$ is positive semidefinite and the diagonal entries of DחD are the same as those of $\mathbf{D}$. Let $\boldsymbol{\Xi}=\operatorname{diag}\left(\xi_{1}, \xi_{2}, \ldots, \xi_{\ell}\right) \triangleq \boldsymbol{\Delta}^{\frac{1}{2}} \boldsymbol{\Pi} \boldsymbol{\Delta}^{\frac{1}{2}}$. It is easy to see that the diagonal entries of DחD are the same as those of $\mathbf{D}$ if and only if the diagonal entries of $(\mathbf{I}-\mathbf{A}) \mathbf{\Xi}(\mathbf{I}-\mathbf{A})$ are the same as those of $\mathbf{I}-\mathbf{A}$. To obtain $\boldsymbol{\Xi}$, we need to solve the linear equations

$$
\left(1-2 a_{i, i}\right) \xi_{i}+\sum_{j=1}^{\ell} a_{i, j}^{2} \xi_{j}=1-a_{i, i}, \quad i=1,2, \ldots, \ell
$$

which can be written compactly as

$$
((\mathbf{I}-\mathbf{A}) \odot(\mathbf{I}-\mathbf{A})) \boldsymbol{\xi}=\mathbf{1}-\mathbf{a}
$$

where $\boldsymbol{\xi}=\left(\xi_{1}, \xi_{2}, \ldots, \xi_{\ell}\right)^{T}$ and $\mathbf{a}=\left(a_{1,1}, a_{2,2}, \ldots, a_{\ell, \ell}\right)^{T}$ Since $(\mathbf{I}-\mathbf{A}) \odot(\mathbf{I}-\mathbf{A}) \rightarrow \mathbf{I}$ and $\mathbf{1}-\mathbf{a} \rightarrow \mathbf{1}$ as $\|\mathbf{d}\| \rightarrow 0$, it follows that $\xi \rightarrow \mathbf{1}$ as $\|\mathbf{d}\| \rightarrow 0$. Therefore, $\boldsymbol{\Pi}-\left(\mathbf{D}+\boldsymbol{\Sigma}_{\mathbf{N}}\right)^{-1}=$ $\boldsymbol{\Delta}^{-\frac{1}{2}} \boldsymbol{\Xi} \boldsymbol{\Delta}^{-\frac{1}{2}}-\left(\mathbf{D}+\boldsymbol{\Sigma}_{\mathbf{N}}\right)^{-1}$ is positive definite when $\|\mathrm{d}\|$ is sufficiently small. The proof is complete. 


\section{APPENDIX E \\ PROOF OF LEMMA 6}

Suppose both $\mathbf{A}$ and $\mathbf{B}$ are diagonal matrices, where

$$
\begin{aligned}
& \mathbf{A} \triangleq \mathbf{D}+\mathbf{\Sigma}_{\mathbf{N}} \\
& \mathbf{B} \triangleq \mathbf{D}^{-1}+\mathbf{\Sigma}_{\mathbf{N}}^{-1} .
\end{aligned}
$$

It is clear that $\mathbf{A}$ and $\mathbf{B}$ are positive definite. Let $\mathbf{D}^{\prime}=$ $\mathbf{A}^{-\frac{1}{2}} \mathbf{D} \mathbf{A}^{-\frac{1}{2}}$ and $\boldsymbol{\Sigma}_{\mathbf{N}}^{\prime}=\mathbf{A}^{-\frac{1}{2}} \boldsymbol{\Sigma}_{\mathbf{N}} \mathbf{A}^{-\frac{1}{2}}$. We have

$$
\begin{aligned}
\mathbf{D}^{\prime}+\boldsymbol{\Sigma}_{\mathbf{N}}^{\prime} & =\mathbf{I} \\
\left(\mathbf{D}^{\prime}\right)^{-1}+\left(\boldsymbol{\Sigma}_{\mathbf{N}}^{\prime}\right)^{-1} & =\mathbf{A}^{\frac{1}{2}} \mathbf{B} \mathbf{A}^{\frac{1}{2}} .
\end{aligned}
$$

Now let $\mathbf{Z}=\left(\mathbf{D}^{\prime}\right)^{-1}-\mathbf{I}$. Note that

$$
\begin{aligned}
\mathbf{Z} & =\left(\mathbf{I}-\mathbf{A}^{-\frac{1}{2}} \boldsymbol{\Sigma}_{\mathbf{N}} \mathbf{A}^{-\frac{1}{2}}\right)^{-1}-\mathbf{I} \\
& =\mathbf{A}^{\frac{1}{2}} \mathbf{D}^{-1} \mathbf{A}^{\frac{1}{2}}-\mathbf{I} \\
& =\mathbf{A}^{\frac{1}{2}}\left(\mathbf{D}^{-1}-\left(\mathbf{D}+\boldsymbol{\Sigma}_{\mathbf{N}}\right)^{-1}\right) \mathbf{A}^{\frac{1}{2}} \\
& =\mathbf{A}^{\frac{1}{2}} \mathbf{D}^{-1}\left(\mathbf{D}^{-1}+\boldsymbol{\Sigma}_{\mathbf{N}}^{-1}\right)^{-1} \mathbf{D}^{-1} \mathbf{A}^{\frac{1}{2}},
\end{aligned}
$$

which implies that $\mathbf{Z}$ is positive definite. Moreover, we have

$$
\begin{aligned}
\mathbf{Z}+\mathbf{Z}^{-1} & =\left(\mathbf{D}^{\prime}\right)^{-1}-\mathbf{I}+\left(\left(\mathbf{D}^{\prime}\right)^{-1}-\mathbf{I}\right)^{-1} \\
& =\left(\mathbf{D}^{\prime}\right)^{-1}+\left(\mathbf{I}-\mathbf{D}^{\prime}\right)^{-1}-2 \mathbf{I} \\
& =\left(\mathbf{D}^{\prime}\right)^{-1}+\left(\boldsymbol{\Sigma}_{\mathbf{N}}^{\prime}\right)^{-1}-2 \mathbf{I} \\
& =\mathbf{C}
\end{aligned}
$$

where $\mathbf{C}=\mathbf{A}^{\frac{1}{2}} \mathbf{B} \mathbf{A}^{\frac{1}{2}}-2 \mathbf{I}$ is a diagonal matrix. Let $\mathbf{Z}=$ $\mathbf{V} \boldsymbol{\Lambda} \mathbf{V}^{T}$ be the eigenvalue decomposition of $\mathbf{Z}$, where $\mathbf{V}$ is a unitary matrix and $\boldsymbol{\Lambda}=\operatorname{diag}\left(\lambda_{1}, \lambda_{2}, \ldots, \lambda_{\ell}\right)$ is a positive definite diagonal matrix. It follows by (33) that

$$
\mathbf{V}\left(\boldsymbol{\Lambda}+\boldsymbol{\Lambda}^{-1}\right) \mathbf{V}^{T}=\mathbf{C} .
$$

Since the eigenvalues of a diagonal matrix are its diagonal entries, there must exist a permutation matrix $\mathbf{P}$ such that $\mathbf{P}^{T}(\boldsymbol{\Lambda}+$ $\left.\boldsymbol{\Lambda}^{-1}\right) \mathbf{P}=\mathbf{C}$. Therefore, we have

$$
\mathbf{V}^{\prime}\left(\boldsymbol{\Lambda}+\boldsymbol{\Lambda}^{-1}\right)\left(\mathbf{V}^{\prime}\right)^{T}=\boldsymbol{\Lambda}+\boldsymbol{\Lambda}^{-1},
$$

where $\mathbf{V}^{\prime}=\left(v_{i, j}^{\prime}\right) \triangleq \mathbf{P V}$ is a unitary matrix. Denote $\boldsymbol{\Lambda}+\boldsymbol{\Lambda}^{-1}$ by $\operatorname{diag}\left(\mu_{1}, \mu_{2}, \ldots, \mu_{\ell}\right)$. It follows by (34) that

$$
\mu_{i} v_{i, j}^{\prime}=\mu_{j} v_{i, j}^{\prime}, \quad i, j=1,2, \ldots, \ell .
$$

Now partition $\{1,2, \ldots, \ell\}$ into disjoint equivalent classes $A_{1}, A_{2}, \ldots, A_{K}$ such that $i \in A_{k}$ and $j \in A_{k}$ for some $k$ if and only if $\mu_{i}=\mu_{j}$. In view of (35), we must have $v_{i, j}^{\prime}=0$ if $i \in \mathrm{A}_{k}$ and $j \in \mathrm{A}_{k^{\prime}}$ with $k \neq k^{\prime}$. As a consequence, if $K \geq 2$, then $\mathbf{D}$ can be converted to a block diagonal matrix (with more than one block) via permutation, which is impossible under our assumption. Therefore, $\{1,2, \ldots, \ell\}$ contains only one equivalent class, which implies $\boldsymbol{\Lambda}+\boldsymbol{\Lambda}^{-1}=\mu \mathbf{I}$ for some $\mu$. So we have $\lambda_{i}=\lambda$ or $\lambda_{i}=\lambda^{-1}$, where $\lambda$ is a solution to
It is clear that we need $\mu>2$ in order for $\lambda$ to be positive. Let $\mathbf{Q}$ be a permutation matrix such that $\boldsymbol{\Lambda}=\mathbf{Q} \operatorname{diag}\left(r: \lambda, \lambda^{-1}\right) \mathbf{Q}^{T}$. We have

$$
\begin{aligned}
\mathbf{D} & =\mathbf{A}^{\frac{1}{2}} \mathbf{D}^{\prime} \mathbf{A}^{\frac{1}{2}} \\
& =\mathbf{A}^{\frac{1}{2}}(\mathbf{I}+\mathbf{Z})^{-1} \mathbf{A}^{\frac{1}{2}} \\
& =\mathbf{A}^{\frac{1}{2}}\left(\mathbf{I}+\mathbf{V} \mathbf{\Lambda} \mathbf{V}^{T}\right)^{-1} \mathbf{A}^{\frac{1}{2}} \\
& =\mathbf{A}^{\frac{1}{2}}\left(\mathbf{I}+\mathbf{V Q} \operatorname{diag}\left(r: \lambda, \lambda^{-1}\right) \mathbf{Q}^{T} \mathbf{V}^{T}\right)^{-1} \mathbf{A}^{\frac{1}{2}} \\
& =\mathbf{A}^{\frac{1}{2}} \mathbf{U} \operatorname{diag}\left(r:(1+\lambda)^{-1},\left(1+\lambda^{-1}\right)^{-1}\right) \mathbf{U}^{T} \mathbf{A}^{\frac{1}{2}},
\end{aligned}
$$

where $\mathbf{U}=\mathbf{V Q}$ is a unitary matrix.

Conversely, if $\mathbf{D}$ can be written in the form of (14), then we can choose

$$
\boldsymbol{\Sigma}_{\mathbf{N}}=\mathbf{A}^{\frac{1}{2}} \mathbf{U} \operatorname{diag}\left(r:\left(1+\lambda^{-1}\right)^{-1},(1+\lambda)^{-1}\right) \mathbf{U}^{T} \mathbf{A}^{\frac{1}{2}} .
$$

It is clear that $\mathbf{D}$ and $\boldsymbol{\Sigma}_{\mathbf{N}}$ are positive definite. Moreover, it can be verified that both $\mathbf{D}+\boldsymbol{\Sigma}_{\mathbf{N}}$ and $\mathbf{D}^{-1}+\boldsymbol{\Sigma}_{\mathbf{N}}^{-1}$ are diagonal matrices. The proof is complete.

\section{APPENDIX F \\ PROOF OF THEOREM 7}

In view of Lemma 6 , if $\mathbf{D}$ satisfies Property 1), then there exists a positive definite matrix $\boldsymbol{\Sigma}_{\mathbf{N}}$ such that both $\mathbf{D}+\boldsymbol{\Sigma}_{\mathbf{N}}$ and $\mathbf{D}^{-1}+\boldsymbol{\Sigma}_{\mathbf{N}}^{-1}$ are diagonal matrices. Indeed, setting

$$
\boldsymbol{\Sigma}_{\mathbf{N}}=\mathbf{A}^{\frac{1}{2}} \mathbf{U} \operatorname{diag}\left(r:\left(1+\lambda^{-1}\right)^{-1},(1+\lambda)^{-1}\right) \mathbf{U}^{T} \mathbf{A}^{\frac{1}{2}}
$$

we can verify that

$$
\begin{aligned}
\mathbf{D}+\boldsymbol{\Sigma}_{\mathbf{N}} & =\mathbf{A} \\
\mathbf{D}^{-1}+\boldsymbol{\Sigma}_{\mathbf{N}}^{-1} & =\left(2+\lambda+\lambda^{-1}\right) \mathbf{A}^{-1}
\end{aligned}
$$

and both of them are diagonal matrices. Moreover, $\boldsymbol{\Sigma}_{\mathbf{X}}^{-1}-\mathbf{D}^{-1}$ is a diagonal matrix due to the fact that $\mathbf{D} \in \mathbb{D}\left(\mathbf{d}, \boldsymbol{\Sigma}_{\mathbf{X}}\right)$. As a consequence, $\boldsymbol{\Sigma}_{\mathbf{X}}^{-1}+\boldsymbol{\Sigma}_{\mathbf{N}}^{-1}$ is also a diagonal matrix since it can be written as

$$
\boldsymbol{\Sigma}_{\mathbf{X}}^{-1}+\boldsymbol{\Sigma}_{\mathbf{N}}^{-1}=\left(\boldsymbol{\Sigma}_{\mathbf{X}}^{-1}-\mathbf{D}^{-1}\right)+\left(\mathbf{D}^{-1}+\boldsymbol{\Sigma}_{\mathbf{N}}^{-1}\right) .
$$

Therefore, we have $\boldsymbol{\Sigma}_{\mathbf{N}} \in \mathbb{N}\left(\boldsymbol{\Sigma}_{\mathbf{X}}\right)$.

In view of Lemma 5, we just need to find a positive semidefinite diagonal matrix $\Delta$ such that the diagonal entries of $\mathbf{D} \Delta \mathbf{D}$ are the same as those of $\left(\mathbf{D}^{-1}+\boldsymbol{\Sigma}_{\mathbf{N}}^{-1}\right)^{-1}$. Note that

$$
\begin{aligned}
\mathbf{D} \boldsymbol{\Delta} \mathbf{D}= & \mathbf{A}^{\frac{1}{2}} \mathbf{U} \operatorname{diag}\left(r:(1+\lambda)^{-1}\right. \\
& \left.\left(1+\lambda^{-1}\right)^{-1}\right) \mathbf{U}^{T} \mathbf{A}^{\frac{1}{2}} \\
& \boldsymbol{\Delta} \mathbf{A}^{\frac{1}{2}} \mathbf{U} \operatorname{diag}\left(r:(1+\lambda)^{-1}\right. \\
& \left.\left(1+\lambda^{-1}\right)^{-1}\right) \mathbf{U}^{T} \mathbf{A}^{\frac{1}{2}} \\
\left(\mathbf{D}^{-1}+\boldsymbol{\Sigma}_{\mathbf{N}}^{-1}\right)^{-1}= & \left(2+\lambda+\lambda^{-1}\right)^{-1} \mathbf{A} .
\end{aligned}
$$

Therefore, after scaling, it suffices to find a positive semidefinite diagonal matrix $\boldsymbol{\Delta}$ such that the diagonal entries of

$\mathbf{U d i a g}\left(r:(1+\lambda)^{-1},\left(1+\lambda^{-1}\right)^{-1}\right) \mathbf{U}^{T}$

$$
\mathbf{\Delta} \mathbf{U d i a g}\left(r:(1+\lambda)^{-1},\left(1+\lambda^{-1}\right)^{-1}\right) \mathbf{U}^{T}
$$

$$
\lambda+\lambda^{-1}=\mu .
$$

are all equal to one. The proof is complete. 


\section{APPENDIX G \\ PROOF OF THEOREM 8}

Here we only prove Part 1) since Part 2) is similar.

We shall show that $\mathbf{D}$ can be written in the form of (14) with $r=1$. It suffices to show that $\mathbf{D}^{-1}$ can be written as

$$
\mathbf{D}^{-1}=\mathbf{A}^{-\frac{1}{2}} \mathbf{U d i a g}\left(1: 1+\lambda, 1+\lambda^{-1}\right) \mathbf{U}^{T} \mathbf{A}^{-\frac{1}{2}}
$$

for some positive definite diagonal matrix $\mathbf{A}=$ $\operatorname{diag}\left(a_{1}, a_{2}, \ldots, a_{\ell}\right)$, unitary matrix $\mathbf{U}=\left(u_{i, j}\right)$, and positive number $\lambda$.

Note that

$$
\begin{aligned}
& \mathbf{A}^{-\frac{1}{2}} \mathbf{U d i a g}\left(1: 1+\lambda, 1+\lambda^{-1}\right) \mathbf{U}^{T} \mathbf{A}^{-\frac{1}{2}} \\
&=\left(1+\lambda^{-1}\right) \mathbf{A}^{-1}-\left(\lambda^{-1}-\lambda\right) \boldsymbol{\eta} \boldsymbol{\eta}^{T}
\end{aligned}
$$

where $\boldsymbol{\eta}=\left(\eta_{1}, \eta_{2}, \ldots, \eta_{\ell}\right)^{T}$ is the first column of $\mathbf{A}^{-\frac{1}{2}} \mathbf{U}$. In view of (18), we can set

$$
\begin{aligned}
\left(1+\lambda^{-1}\right) \mathbf{A}^{-1} & =\Delta_{\mathbf{D}}^{-1} \\
\sqrt{\lambda^{-1}-\lambda} \boldsymbol{\eta} & =\boldsymbol{\beta} .
\end{aligned}
$$

As a consequence, we have

$$
u_{i, 1}=a_{i}^{\frac{1}{2}} \eta_{i}=\frac{\beta_{i} \delta_{\mathbf{D}, i}^{\frac{1}{2}}}{\sqrt{1-\lambda}}, \quad i=1,2, \ldots, \ell .
$$

Since $\mathbf{U}$ has to be a unitary matrix, we must have

$$
\sum_{i=1}^{\ell} u_{i, 1}^{2}=1
$$

which implies

$$
\lambda=1-\sum_{i=1}^{\ell} \beta_{i}^{2} \delta_{\mathbf{D}, i}
$$

In view of (16) and (18), one can readily show that $\lambda \in(0,1] .^{3}$ Since the right-hand side (RHS) of (36) depends on $\mathbf{U}$ only through its first column, our construction shows that $\mathbf{D}^{-1}$ can indeed be written in the form of (36).

Now by Theorem 7, it suffices to have a positive semidefinite diagonal matrix $\Delta$ such that the diagonal entries of

$\mathbf{U d i a g}\left(1:(1+\lambda)^{-1},\left(1+\lambda^{-1}\right)^{-1}\right) \mathbf{U}^{T}$

$$
\boldsymbol{\Delta} \mathbf{U} \operatorname{diag}\left(1:(1+\lambda)^{-1},\left(1+\lambda^{-1}\right)^{-1}\right) \mathbf{U}^{T}
$$

are all equal to one. Note that

$$
\begin{aligned}
\mathbf{U d i a g}\left(1:(1+\lambda)^{-1},\left(1+\lambda^{-1}\right)^{-1}\right) & \mathbf{U}^{T} \\
& =\left(1+\lambda^{-1}\right)^{-1}\left(\mathbf{I}+\mathbf{v} \mathbf{v}^{T}\right)
\end{aligned}
$$

where $\mathbf{v}=\left(v_{1}, v_{2}, \ldots, v_{\ell}\right)^{T}$ with

$$
\begin{aligned}
v_{i}=\sqrt{\lambda^{-1}-1} u_{i, 1}=\frac{\beta_{i} \delta_{\mathbf{D}, i}^{\frac{1}{2}}}{\sqrt{1-\sum_{j=1}^{l} \beta_{j}^{2} \delta_{\mathbf{D}, j}}} \\
i=1,2, \ldots, \ell .
\end{aligned}
$$

${ }^{3}$ If $\lambda=1$, then $\mathbf{U}$ can be an arbitary unitary matrix. Note that $\lambda=1$ if and only if $\alpha_{i}=0$ for all $i$.
Therefore, we just need to have a positive semidefinite diagonal matrix $\boldsymbol{\Delta}=\operatorname{diag}\left(\delta_{1}, \delta_{2}, \ldots, \delta_{\ell}\right)$ such that the diagonal entries of $\left(\mathbf{I}+\mathbf{v} \mathbf{v}^{T}\right) \boldsymbol{\Delta}\left(\mathbf{I}+\mathbf{v} \mathbf{v}^{T}\right)$ are all equal to one, i.e., the solution $\boldsymbol{\delta}=\left(\delta_{1}, \delta_{2}, \ldots, \delta_{\ell}\right)^{T}$ to the linear equations

$$
\left(1+2 v_{i}^{2}\right) \delta_{i}+v_{i}^{2} \sum_{j=1}^{\ell} v_{j}^{2} \delta_{j}=1, \quad i=1,2, \ldots, \ell
$$

satisfies $\delta_{i} \geq 0, i=1,2, \ldots, \ell$. Note that (37) can be rewritten as

$$
\left(\Phi+\zeta \zeta^{T}\right) \boldsymbol{\delta}=1
$$

where $\boldsymbol{\Phi}=\operatorname{diag}\left(1+2 v_{1}^{2}, 1+2 v_{2}^{2}, \ldots, 1+2 v_{\ell}^{2}\right)$ and $\zeta=$ $\left(v_{1}^{2}, v_{2}^{2}, \ldots, v_{\ell}^{2}\right)^{T}$. It follows by (15) in Lemma 7 that

$$
\begin{aligned}
\boldsymbol{\delta} & =\left(\boldsymbol{\Phi}+\zeta \zeta^{T}\right)^{-1} \mathbf{1} \\
& =\left(\boldsymbol{\Phi}^{-1}-\frac{1}{1+\sum_{j=1}^{\ell} \frac{v_{j}^{4}}{1+2 v_{j}^{2}}} \zeta^{\prime}\left(\boldsymbol{\zeta}^{\prime}\right)^{T}\right) \mathbf{1}
\end{aligned}
$$

where $\zeta^{\prime}=\left(\frac{v_{1}^{2}}{1+2 v_{1}^{2}}, \frac{v_{2}^{2}}{1+2 v_{2}^{2}}, \ldots, \frac{v_{\ell}^{2}}{1+2 v_{\ell}^{2}}\right)^{T}$. As a consequence, we have

$$
\delta_{i}=\frac{1+\sum_{j=1}^{\ell} \frac{v_{j}^{2}\left(v_{j}^{2}-v_{i}^{2}\right)}{1+2 v_{j}^{2}}}{\left(1+2 v_{i}^{2}\right)\left(1+\sum_{j=1}^{\ell} \frac{v_{j}^{4}}{1+2 v_{j}^{2}}\right)}, \quad i=1,2, \ldots, \ell .
$$

It is clear that $\delta_{i} \geq 0$ if and only if

$$
1+\sum_{j=1}^{\ell} \frac{v_{j}^{2}\left(v_{j}^{2}-v_{i}^{2}\right)}{1+2 v_{j}^{2}} \geq 0
$$

The proof is complete.

\section{REFERENCES}

[1] T. Berger, "Multiterminal source coding," in The Information Theory Approach to Communications, G. Longo, Ed. New York: SpringerVerlag, 1978, vol. 229, pp. 171-231, CISM Courses and Lectures.

[2] T. Berger, Z. Zhang, and H. Viswanathan, "The CEO problem," IEEE Trans. Inf. Theory, vol. 42, pp. 887-902, May 1996.

[3] S. Boyd and L. Vandenberghe, Convex Optimization. Cambridge, U.K.: Cambridge Univ. Press, 2004.

[4] S. N. Diggavi and T. M. Cover, "The worst additive noise under a covariance constraint," IEEE Trans. Inf. Theory, vol. 47, pp. 3072-3081, Nov. 2001.

[5] Y. Oohama, "Gaussian multiterminal source coding," IEEE Trans. Inf. Theory, vol. 43, pp. 1912-1923, Nov. 1997.

[6] Y. Oohama, "The rate-distortion function for the quadratic Gaussian CEO problem," IEEE Trans. Inf. Theory, vol. 44, pp. 1057-1070, May 1998.

[7] Y. Oohama, "Rate-distortion theory for Gaussian multiterminal source coding systems with several side informations at the decoder," IEEE Trans. Inf. Theory, vol. 51, pp. 2577-2593, Jul. 2005.

[8] Y. Oohama, "Gaussian multiterminal source coding with several side informations at the decoder," in Proc. IEEE Int. Symp. Inf. Theory, Jul. 2006, pp. 1409-1413.

[9] Y. Oohama, "Rate distortion region for distributed source coding of correlated Gaussian remote sources," in Proc. IEEE Int. Symp. Inf. Theory, Jul. 2008, pp. 41-45.

[10] Y. Oohama, "Multiterminal source coding problem with several side informations at the decoder," in Proc. IEEE Int. Symp. Inf. Theory, Jul. 2008, pp. 687-691.

[11] Y. Oohama, "Distributed source coding of correlated Gaussian observations," in Proc. 2008 Int. Symp. Inf. Theory Appl., Auckland, New Zealand, Dec. 7-10, 2008, pp. 1441-1446. 
[12] Y. Oohama, Many-Help-One Problem for Gaussian Sources With a Tree Structure on Their Correlation [Online]. Available: http://arxiv. org/abs/0901.1988

[13] V. Prabhakaran, D. Tse, and K. Ramchandran, "Rate region of the quadratic Gaussian CEO problem," in Proc. IEEE Int. Symp. Inf. Theory, 2004, p. 117.

[14] J. Rohn, "An existence theorem for systems of nonlinear equations," ZAMM, vol. 60, p. 345, 1980, Kleine Mitteilungen/Buchbesprechungen.

[15] G. Sansone and R. Conti, Non-Linear Differential Equations. Oxford, U.K.: Pergamon, 1964.

[16] D. Slepian and J. K. Wolf, "Noiseless coding of correlated information sources," IEEE Trans. Inf. Theory, vol. IT-19, pp. 471-480, Jul. 1973.

[17] H. Stark and J. W. Woods, Probability, Random Processes, and Estimation Theory for Engineers. Englewood Cliffs, NJ: Prentice-Hall, 1986.

[18] S. Tavildar and P. Viswanath, "On the sum-rate of the vector Gaussian CEO problem," in Proc. 39th Asilomar Conf. Signals, Syst. Comput., 2005, pp. 3-7.

[19] S. Tavildar, P. Viswanath, and A. B. Wagner, "The Gaussian manyhelp-one distributed source coding problem," IEEE Trans. Inf. Theory, vol. 56, pp. 564-581, Jan. 2010.

[20] S.-Y. Tung, "Multiterminal Source Coding," Ph.D. dissertation, Cornell Univ., School of Elect. Eng., Ithaca, NY, 1978.

[21] H. Viswanathan and T. Berger, "The quadratic Gaussian CEO problem," IEEE Trans. Inf. Theory, vol. 43, pp. 1549-1559, Sep. 1997.

[22] A. B. Wagner, S. Tavildar, and P. Viswanath, "Rate region of the quadratic Gassian two-encoder source-coding problem," IEEE Trans. Inf. Theory, vol. 54, pp. 1938-1961, May 2008.

[23] H. Weingarten, Y. Steinberg, and S. Shamai (Shitz), "The capacity region of the gaussian multiple-input multiple-output broadcast channel," IEEE Trans. Inf. Theory, vol. 52, pp. 3936-3964, Sep. 2006.

[24] A. Shapiro, "Duality, optimality conditions, and perturbation analysis," in Handbook of Semidefinite Programming: Theory, Algorithms, and Applications, H. Wolkowicz, R. Saigal, and L. Vandenberghe, Eds. Norwell, MA: Kluwer, 2000, pp. 68-92.

[25] R. Zamir and T. Berger, "Multiterminal source coding with high resolution," IEEE Trans. Inf. Theory, vol. 45, no. 1, pp. 106-117, Jan. 1999.
Jia Wang received the B.Sc. degree in electrical engineering, the M.S. degree in pattern recognition and intelligence control, and the Ph.D. degree in electrical engineering from Shanghai Jiao Tong University, China, in 1997, 1999, and 2002, respectively.

Since 2002, he has been with the Institute of Image Communication and Information Processing, where he is currently an Associate Professor. His research interests include multiuser information theory and its application in video coding.

Jun Chen (S'03-M'06) received the B.E. degree with honors in communication engineering from Shanghai Jiao Tong University, Shanghai, China, in 2001, and the M.S. and Ph.D. degrees in electrical and computer engineering from Cornell University, Ithaca, NY, in 2004 and 2006, respectively.

He was a Postdoctoral Research Associate with the Coordinated Science Laboratory, University of Illinois at Urbana-Champaign, Urbana, from 2005 to 2006, and a Postdoctoral Fellow with the IBM Thomas J. Watson Research Center, Yorktown Heights, NY, from 2006 to 2007. He is currently an Assistant Professor of Electrical and Computer Engineering at McMaster University, Hamilton, ON, Canada. He holds the Barber-Gennum Chair in Information Technology. His research interests include information theory, wireless communications, and signal processing.

Dr. Chen received the Josef Raviv Memorial Postdoctoral Fellowship in 2006 and the Early Research Award from the Province of Ontario in 2010.

Xiaolin Wu (M'89-SM'96) received the B.Sc. degree from Wuhan University, China, in 1982, and the Ph.D. degree from the University of Calgary, AB, Canada, in 1988, both in computer science.

He started his academic career in 1988, and has since been on the faculty of the University of Western Ontario, New York Polytechnic University, and currently McMaster University, Hamilton, ON, Canada, where he is a Professor with the Department of Electrical and Computer Engineering and holds the NSERC-DALSA Industrial Research Chair in Digital Cinema. His research interests include multimedia signal compression, joint source-channel coding, multiple description coding, network-aware visual communication, and image processing. He has published more than 200 research papers and holds two patents in these fields.

Dr. Wu is an Associate Editor of IEEE TRANSACTIONS ON MULTIMEDIA and an Associate Editor of IEEE TRANSACTIONS ON IMAGE PROCESSING. 